\title{
Picard Groups of Hypersurfaces in Toric Varieties
}

\author{
By \\ Shi-shyr ROAN*
}

\begin{abstract}
We study the structure of rational Picard groups of hypersurfaces of toric varieties. By using the fan structure associated to the ambient toric varrety, an explicit basis of the Picard group is described by certain combinatorial data. We shall also discuss the application to Calabi-Yau spaces.
\end{abstract}

\section{\$1. Introduction}

Throughout this paper by an orbifold we shall mean a complex variety with at worst abelian quotient singularities. The orbifolds we shall study here are toric varieties and their quasi-smooth hypersurfaces. Much of what was needed in toric geometry is already in [7] [10] [12] [15] [16], and the present paper borrows heavily from those references. The purpose of this paper is to determine the (rational) Picard groups, i.e. the groups of $\mathbf{Q}$-divisors, of hypersurfaces in toric varieties. The quantitative aspect of Picard groups, as well as the qualitative one, will be the main concern of this work. In algebraic geometry, the toric divisors provide a combinatorial description for the structure of Q-divisors of a toric variety [5] [14]. However, one can also study this subject from sympletic geometry point of view [3] [11]. As in the case of projective spaces where line bundles are obtained by the induced bundles from Hopf bundle through characters of $\mathbf{C}^{*}$, we can also describe the Picard groups of toric varieties in a similar manner. The present work focuses on the structure on Picard groups of hypersurfaces in toric varieties, which ultimately yields some combinatorial basis-representation for $H^{1,1}$ of Calabi-Yau (CY) mirror 3-folds. There has been considerable progress on this subject over the last few years [26]: the special case on quotients of "Fermat-type" CY hypersurfaces in [21]

Communicated by S. Mori, November 17, 1995.

1991 Mathematics Subject Classification (s): 14M35, 32J17

*Institute of Mathematics, Academia Sinica, Taipei, Taiwan.

Supported in part by the NSC grant of Taiwan. 
[22]; and also the general cases but focusing on Picard numbers only in [4]. The goal of this sequel is to show that an investigation of the general properties reveals a remarkably profound structure, and hence to enrich our understanding on toric geometry and mirror symmetry.

The following is a summary of the contents of this paper: Section 2 contains some basic definitions in this work, including orbifold principal bundles with torus as the structure group. The homogeneous coordinate system of toric varieties found by Audin [3] and Cox [6] [18] [2] is the main example for such notion in the present paper. In Section 3, the Picard groups of toric varieties are constructed through the induced bundle-construction which behaves much the same as in the usual projective space. In Section 4 , we aim to give an overview of some basic properties on quasi-smooth hypersurfaces in toric varieties for later use. For the purpose of illustrating the general results in the previous sections, we shall present some standard examples of toric varieties in Section 5 by connecting them with some well-known facts on those varieties. In the next two sections we shall deal with the most significant objects in this paper. In Section 6, Picard groups of hypersurfaces of toric varieties are determined through the related combinatorial data. The hypersurfaces are in general not ample, however we must assume some additional (somewhat unpleasant) conditions on the toric varieties. The prime examples in our mind are CY spaces appeared in the context of mirror symmetry [4] [21] [22] [26], whose structure has led us to this work. In Section 7, we shall apply the results of previous sections to anti-canonical hypersurfaces in the toric varieties of reflexive polytope-type introduced by Batyrev in [4]. With an additional "maximal" condition on the combinatorial data, the hypersurfaces are smooth in the dimension 3. Hence we obtain a combinatorial description of Picard groups for these CY spaces, which generalize the results in [21] [22] on the quotients of Fermat-type CY hypersurfaces. In this paper, we have endeavored to put the presentation in the context of complex geometry in the hope of making easier access to differential geometers and topologists. Some of what is included should be undoubtedly known to experts in toric geometry; nonetheless, it seems difficult to find an appropriate references, especially in Sections 2 and 4 , some subjects we have taken pains to include. We have put some elementary arguments for a few well-known facts of the content in the appendix for easy reference.

\section{Notations}

We prepare some notations for easier presentation of this paper. $A_{\mathbb{K}}=A \otimes_{\mathbb{Z}} \mathbb{K}$, for an abelian group $A$ and a field $\mathbb{K}=\mathbb{Q}, \mathbb{R}, \mathbb{C}$.

For a $n$-dimensional lattice $L$, we denote $\mathbb{T}(L)=L_{\mathbb{C}} / L\left(=\exp \left(2 \pi i L_{\mathbb{C}}\right)\right)$, the $n$-dimensional (algebraic) torus having $L$ 
as the group of one-parameter subgroups.

$L^{*}=\operatorname{Hom}(L, \mathbb{Z})$, the dual lattice of $L$.

$\langle *, *\rangle:, L_{\mathbf{R}} \times L_{\mathbf{R}}^{*} \rightarrow \mathbf{R}$, the non-degenerate natural pairing which takes integral values on $L \times L^{*}$. For a subset $\tau$ of $L_{\mathbf{R}}$, the annihilator of $\tau$ is defined by

$$
\tau^{\perp}:=\left\{y \in L_{\mathbf{R}}^{*} \mid\langle x, y\rangle=0 \forall x \in \tau\right\}
$$

$\Sigma=$ a fan in $L_{\mathbf{R}}$, which will always be a rational simplical fan for the lattice $L$ throughout the paper unless otherwise specified. $\sum^{(i)}$ denotes the $i$-th skeleton of $\sum$.

$\mathbb{P}_{(\Sigma, L)}=$ the $\mathbf{T}(L)$-toric variety associated to a complete fan $\Sigma$, (note that $\mathbb{P}_{(\Sigma, L)}$ is an orbifold by the assumption on $\Sigma$ ).

\section{§2. Orbifold Principal Bundles}

For convenience of later discussions, we introduce the following notion on orbifolds as a generalization of principle bundles and induced line bundles in the class of complex manifolds :

Definition 1. Let $\mathbb{T}$ be a (algebraic) torus. A morphism of orbifolds,

$$
\pi: P \rightarrow X
$$

is called a principal orbifold $\mathbb{T}$-bundle if the following conditions hold:

(i) $P$ is a $\mathbb{T}$-space with the right $\mathbb{T}$-action:

$$
P \times \mathbb{T} \rightarrow P
$$

and $\pi$ is a $\mathbf{T}$-equivariant morphism with the trivial $\mathbf{T}$-structure on $X$.

(ii) For $x \in X$, there is an open neighborhood $U_{x}$ of $x$, and a finite abelian group $G_{x}$ such that

$$
\pi_{\text {rest }}: \pi^{-1}\left(U_{x}\right) \rightarrow U_{x}
$$

is $\mathbb{T}$-equivalent to a left $G_{x}$-quotient of the product bundle,

$$
G_{x} \backslash\left(B_{\epsilon}(0) \times \mathbf{T} \stackrel{p r}{\rightarrow} B_{\epsilon}(0)\right), B_{\epsilon}(0):=\left\{z \in \mathbb{C}^{n} ;|z|<\epsilon\right\}
$$

where the $G_{x}$-action is given by 


$$
G_{x} \times B_{\epsilon}(0) \times \mathbb{T} \rightarrow B_{\epsilon}(0) \times \mathbb{T},(g,(z, t)) \mapsto g \cdot(z, t)=(g \cdot z, \varphi(g) t),
$$

for some group homomorphism $\varphi: G_{x} \rightarrow \mathbb{T}$.

(iii) $G_{x}$ is the trivial group for a non-singular point $x$.

Note that $\mathbb{T}$ acts on $\pi^{-1}(x)$ transitively for each $x \in X$. Hence all elements of $\pi^{-1}(x)$ have the same finite isotropy subgroup of $\mathbb{T}$, denoted by $g_{x}$. For $U_{x}$ in (ii) of the above definition, an element $y$ of $U_{x}$ corresponds to a $G_{x}$-orbit $\left[z_{0}\right]$ for some $z_{0} \in \mathbb{C}^{n}$. We have the following isomorphic germs of analytic spaces:

$$
\left(U_{x}, y\right) \simeq\left(\left\{z \in \mathbb{C}^{n}|| z-z_{0} \mid<\delta\right\} / G_{x, y},\left[z_{0}\right]\right),
$$

where $G_{x, y}$ is the subgroup of $G_{x}$ stablizing the fiber over $z_{0}$, ( note that the group $G_{x, y}$ does not depend on the choice of $z_{0}$ ). Then we have a surjective homomorphism

$$
G_{x, y} \rightarrow g_{y}, \quad \text { for } y \in U_{x}
$$

Definition 2. For a principal orbifold $\mathbb{T}$-bundle,

$$
\pi: P \rightarrow X
$$

and a character of $\mathbb{T}$,

$$
\chi: \mathbb{T} \rightarrow \mathbb{C}^{*},
$$

$P \times_{\mathbb{T}} \mathbb{C}$ is the quotient space of $P \times \mathbb{C}$ by the $\mathbb{T}$-action:

$$
(p, \zeta) \cdot t=(p \cdot t, \chi(t) \zeta)
$$

The fibration

$$
P \times_{\mathbb{T}} \mathbb{C} \rightarrow X,[(p, \zeta)] \mapsto \pi(p),
$$

will be denoted by $\mathscr{O}(\chi)$, called the orbifold line bundle induced by $\chi^{-1}$.

Note that $\mathscr{O}(\chi)$ is a line bundle outside the singular set of $X$. The sections of $\mathscr{O}(\chi)$ over an open set $U$ of $X$ can be regarded as functions on $\pi^{-1}(U)$. In fact, one has

$$
\Gamma(U, \mathscr{O}(\chi))=\left\{f: \pi^{-1}(U) \rightarrow \mathbb{C} \mid f(p \cdot t)=\chi(t) f(p) \quad \text { for } p \in \pi^{-1}(U), t \in \mathbb{T}\right\}
$$


We shall make this identification in what follows. For compact $X, \mathscr{O}(\chi)$ is considered as an element of the rational Picard group of $X$ by the following lemma:

Lemma 1. For a compact variety $X, \mathscr{O}\left(\chi^{N}\right)$ is a line bundle over $X$ for some positive integer $N$.

Proof. Let $U_{x}, G_{x}$ be the same as in the condition (ii) of Definition 1. By the compactness of $X$, there is a positive interger $N$ divisible by $\left|G_{x}\right|$ for all $x \in$ $X$. Claim: $\mathscr{O}\left(\chi^{N}\right)$ is a line bundle which is trivial over $U_{x}$. By the construction of orbifold line bundle, $\mathscr{O}\left(\chi^{N}\right)_{\mid U x}$ is equivalent to

$$
G_{x} \backslash\left(B_{\epsilon}(0) \times \mathbb{C} \stackrel{p r}{\rightarrow} B_{\epsilon}(0)\right)
$$

with the $G_{x}$-action given by

$$
\begin{aligned}
g \cdot(z, \zeta) & =\left(g \cdot z, \chi^{N}(g \cdot 1) \zeta\right) \\
& =(g \cdot z, \zeta), \quad\left(\text { by }\left|G_{x}\right| \mid N\right)
\end{aligned}
$$

Therefore $\mathscr{O}\left(\chi^{N}\right)_{U_{x}}$ is isomorphic to $U_{x} \times \mathbb{C}$.

Let $\mathbb{P}_{(\Sigma, L)}$ be the toric variety for a $n$-dimensional lattice $L$ and a complete fan $\sum$ in $L_{\mathbf{R}}$. By [3] [6], $\mathbf{P}_{(\Sigma, L)}$ can be regarded as the orbit-space for some torus action on a "homogeneous" affine variety. We are going to show that this fibration over $\mathbf{P}_{(\Sigma, L)}$ is in fact a principal orbifold bundle. The "homogeneous" affine variety will be described through the toric construction as in [18].

Let $\Sigma^{1}$ be the collection of primitive elements in $L$ which are the generators of elements in 1-skeleton $\Sigma^{(1)}$. For a simplicial cone $\sigma$ of $\Sigma$, the simplex in $L_{\mathbb{R}}$ spanned by $\Sigma^{1} \cap \sigma$ and the origin is denoted by $\Delta_{\sigma}$. Consider the (compact) polytope in $L_{\mathbf{R}}$,

$$
\Delta(L):=\bigcup_{\sigma \in \Sigma} \Delta_{\sigma}
$$

The faces of $\Delta_{\sigma}$ 's form a (simplicial) triangulation of $\Delta(L)$, denoted by $\left\{s_{t}\right\}_{i \in I}$, with $\Sigma^{1} \cup\{0\}$ as the set of 0 -simplices. Hence the complete fans in $L_{\mathbf{R}}$ are in one-to-one correspondence with the triangulated polytopes $\left(\Delta(L),\left\{s_{\imath}\right\}_{i \in I}\right)$ in $L_{\mathbf{R}}$ such that the following properties hold for 0 -simplices $s_{i}$,

$$
\begin{array}{cl}
s_{i} \in \text { interior } \\
s_{i} \neq \overrightarrow{0} & \Leftrightarrow s_{i}=\text { the origin } \overrightarrow{0},
\end{array}
$$


The toric variety $\mathbb{P}_{(\Sigma, L)}$ is determined by the triangulation of $\Delta(L)$. It is known that the irreducible toric divisors of $\mathbb{P}_{(\Sigma, L)}$ are in one-to-one correspondence with the elements in $\Sigma^{1}$, and denote $e_{\delta}$ the toric divisor corresponding to an element $\delta \in \Sigma^{1}$. Consider the free $\mathbb{Z}$-module $D_{0}$ of rank $d\left(:=\left|\Sigma^{1}\right|\right)$ with the $\mathbb{Z}$-basis given by the set of all irreducible toric divisors of $\mathbb{P}_{(\Sigma, L)}$, and we shall denote $\mathbb{Z}$-basis elements of $D_{0}$ by $e_{\delta}$ again if no confusion could arise,

$$
D_{0}:=\bigoplus_{\delta \in \Sigma^{1}} \mathbb{Z} e_{\delta}
$$

(Note that no linear equivalent relation of divisors exists among $e_{\delta}^{\prime}$ s in $D_{0}$.) Define the homomorphism

$$
\beta_{0}: D_{0} \rightarrow L \text { with } \beta_{0}\left(e_{\delta}\right)=\delta
$$

and denote its kernel by

$$
\mathbb{n}\left(=\mathbb{n}\left(\sum, L\right)\right):=\operatorname{Ker}\left(\beta_{0}\right)
$$

Since the cokernel of $\beta_{0}$ is finite, by tensoring with $\mathbb{R}$, we have the surjective linear map:

$$
\beta=\left(\beta_{0}\right)_{\mathbb{R}}:\left(D_{0}\right)_{\mathbb{R}}=\bigoplus_{\delta \in \Sigma^{1}} \mathbb{R} e_{\tilde{\delta}} \rightarrow L_{\mathbb{R}}
$$

Let $D$ be a lattice in $\oplus_{\delta \in \Sigma^{1}} \mathbb{R} e_{\delta}$ with the following property :

(i) $D \supseteq D_{0}$.

(ii) $\beta(D)=L$, and $\beta$ induces an isomorphism of finite abelian groups:

$$
D / D_{0} \simeq L / L_{0}, \quad \text { with } \quad L_{0}:=\sum_{\delta \in \Sigma^{1}} \mathbb{Z} \delta .
$$

Note that $D$ is equal to $\oplus_{\delta \in \Sigma^{1}} \mathbb{Z}_{e_{\delta}}$ if $\beta_{0}$ is surjective. In general, the lattice $D$ is not uniquely determined. However we simply choose one such lattice for our purpose and denote it by $D(=D(\Sigma, L))$. Then we have the exact sequence of abelian groups :

$$
0 \rightarrow \mathbb{n} \stackrel{\iota}{\rightarrow} D \stackrel{\beta}{\rightarrow} L \rightarrow 0
$$

and its dual sequence:

$$
0 \rightarrow L^{*} \stackrel{\beta^{*}}{\rightarrow} D^{*} \stackrel{\iota^{*}}{\rightarrow} \mathbb{R}^{*} \rightarrow 0
$$


Regard (4) and (5) as the corresponding 1-parameter subgroups and characters respectively for the exact sequence of tori:

$$
0 \rightarrow \mathbb{T}(\mathbf{n}) \rightarrow \mathbb{T}(D) \rightarrow \mathbf{T}(L) \rightarrow 0
$$

and identify the following vector spaces:

$$
D_{\mathbf{K}}=\left(D_{0}\right)_{\mathbf{K}}=\bigoplus_{\delta \in \Sigma^{1}} \mathbf{K} e_{\delta}, \quad \text { for } \mathbf{K}=\mathbf{Q}, \mathbf{R}, \mathbf{C}
$$

Denote

$$
\left\{e_{\delta}^{*}\right\}_{\delta \in \Sigma^{1}}=\text { the basis of } D_{\mathbf{K}}^{*} \text { dual to }\left\{e_{\delta}\right\}_{\delta \in \Sigma^{1}}
$$

For an element $\sigma$ in a fan $\sum$ in $L_{\mathbb{R}}$, let $\widetilde{\sigma}$ be the simplicial cone in $D_{\mathbb{R}}$ defined by

$$
\widetilde{\sigma}:=\sum_{\delta \in \sigma \cap \Sigma^{1}} \mathbb{R}_{\geq 0} e_{\delta} \subset D_{\mathbb{R}},
$$

and $\widetilde{\Sigma}$ be the collection of all such $\widetilde{\sigma}$,

$$
\widetilde{\Sigma}:=\{\widetilde{\sigma} \mid \sigma \in \Sigma\} \text {. }
$$

Then $\widetilde{\Sigma}$ is a fan in $D_{\mathbb{R}}$ with its support $|\widetilde{\Sigma}|$ contained in the boundary of the first quardrant cone $\Omega$ in $D_{\mathbb{R}}$ :

$$
\Omega:=\sum_{\delta \in \Sigma^{1}} \mathbb{R}_{\geq 0} e_{\delta}
$$

Let $\mathbb{C}_{(\Sigma, L)}$ be the $\mathbb{T}(D)$-variety associated to the fan $\widetilde{\Sigma}$. It is an open subset of its closure $\overline{\mathbf{C}_{(\Sigma, L)}}$, which is a $\mathbf{T}(D)$-affine variety :

$$
\mathbb{C}_{(\Sigma, L)} \subset \overline{\mathbb{C}_{(\Sigma, L)}}=\operatorname{Spec} \mathbb{C}\left[\Omega^{*} \cap D^{*}\right]
$$

The above spaces can be realized as orbifolds in the following manner. Associate to the pair $\left(\sum, L_{0}\right)$, one has the $\mathbf{T}\left(D_{0}\right)$-toric variety $\overline{\mathbf{C}_{\left(\Sigma, L_{0}\right)}}$, which is isomorphic to $\mathbb{C}^{d}$ :

$$
\overline{\mathbf{C}_{(\Sigma, L 0)}}=\bigoplus_{\delta \in \Sigma^{1}} \mathbf{C}_{e_{\delta}} \simeq \mathbf{C}^{d},
$$


and

$$
\mathbb{C}_{\left(\Sigma, L_{0}\right)}=\overline{\mathbb{C}_{\left(\Sigma, L_{0}\right)}}-\bigcup_{I}\left\{\sum_{\delta} z_{\delta} e_{\delta} \mid z_{\delta}=0 \text { for } \delta \in I\right\},
$$

where the index $I$ runs over subsets of $\sum^{1}$ with $I \neq \sigma \cap \sum^{1}$ for some $\sigma \in \Sigma$. Since the torus $\mathbb{T}\left(D_{0}\right)$ is a finite cover of $\mathbb{T}(D)$ with the kernel $D / D_{0}$ :

$$
0 \rightarrow D / D_{0} \rightarrow \mathbb{T}\left(D_{0}\right) \rightarrow \mathbb{T}(D) \rightarrow 0
$$

one has the isomorphisms :

$$
\begin{aligned}
& \overline{\mathbb{C}_{(\Sigma, L)}} \simeq \overline{\mathbb{C}_{\left(\Sigma, L_{0}\right)}} /\left(D / D_{0}\right) \simeq \mathbb{C}^{d} /\left(D / D_{0}\right), \\
& \mathbb{C}_{(\Sigma, L)} \simeq \mathbb{C}_{\left(\Sigma, L_{0}\right)} /\left(D / D_{0}\right) .
\end{aligned}
$$

Therefore $\overline{\mathbb{C}_{(\Sigma, L)}}-\mathbb{C}_{(\Sigma, L)}$ is an union of affine subvarieties of $\overline{\mathbb{C}_{(\Sigma, L)}}$ having the codimension $\geq 2$. Since $\mathbb{C}_{(\Sigma, L)}$ is an union of $\mathbb{T}(D)$-orbits, it is stable under the action of $\mathbb{T}(\mathbb{m})$. The linear map $\beta$ of (3) induces a map from $\tilde{\Sigma}$ to $\Sigma$ sending $\widetilde{\sigma}$ to $\sigma$, hence a morphism :

$$
\pi: \mathbb{C}_{(\Sigma, L)} \rightarrow \mathbb{P}_{(\Sigma, L)}
$$

Composing $\pi$ with the finite projection from $\mathbb{C}_{\left(\Sigma, L_{0}\right)}$ to $\mathbb{C}_{(\Sigma, L)}$, one obtains the morphism

$$
\pi_{0}: \mathbb{C}_{\left(\Sigma, L_{0}\right)} \rightarrow \mathbb{P}_{(\Sigma, L)}
$$

The coordinates of the affine spaces $\mathbb{C}_{\left(\Sigma, L_{0}\right)}$ or $\mathbb{C}_{(\Sigma, L)}$ can be regarded as the generalized homogeneous coordinates for the toric variety $\mathbb{P}_{(\Sigma, L)}$ in $[3][6]$ ( see also [11] [18] ). And $\mathbb{P}_{(\Sigma, L)}$ can be considered as the set of $\mathbb{T}(\mathbb{R})$-orbits in $\mathbb{C}_{(\Sigma, L)}$ :

$$
\mathbb{C}_{(\Sigma, L)} / \mathbb{T}(\mathbb{R})=\mathbb{P}_{(\Sigma, L)}
$$

In fact, $\mathbb{C}_{(\Sigma, L)}$ is a principal $\mathbb{T}(\mathbb{R})$-bundle over $\mathbb{P}_{(\Sigma, L)}$ in the sense of Definition 1 .

Lemma 2. With the $\mathbb{T}(\mathbb{R})$-action on $\mathbb{C}_{(\Sigma, L)}$, the fibration $(7)$ is a principle orbifold $\mathbb{T}(\mathbb{R})$ - bundle.

Proof. It is known that $U_{\sigma}\left(=\operatorname{Spec} \mathbb{C}\left[\check{\sigma} \cap L^{*}\right]\right), \sigma \in \Sigma$, form an open affine cover of $\mathbb{P}_{(\Sigma, L)}$, and the $\mathbb{T}(L)$-orbits in $\mathbb{P}_{\Sigma}$ are in an one-to-one correspondence with the open cones $\stackrel{\circ}{\sigma}$ : 


$$
\stackrel{\circ}{\sigma} \leftrightarrow \operatorname{orb}(\sigma), \sigma \in \Sigma
$$

Over $U_{\sigma}, \pi^{-1}\left(U_{\sigma}\right)$ is the affine open subvariety $\mathbf{V}_{\tilde{\sigma}}$ of $\mathbf{C}_{(\Sigma, L)}$,

$$
V_{\tilde{\sigma}}:=\operatorname{Spec} \mathbf{C}\left[\check{\widetilde{\sigma}} \cap D^{*}\right],
$$

and the restriction of $\pi$,

$$
\pi_{\sigma}: V \widetilde{\sigma} \rightarrow U_{\sigma}
$$

is the morphism of toric varieties induced by $\beta$. For $x \in \operatorname{orb}(\sigma), U_{\sigma}$ is an open neighborhood of $x$. Let $\left\{\delta_{j}\right\}_{j=1}^{m}$ be the intersection of $\sum^{1}$ with $\sigma$, and $L_{\sigma}$ denotes the sublattice $L \cap \sum_{j=1}^{m} \mathbb{R} \delta_{j}$ of $L$. Let $\rho_{k},(1 \leq k \leq n-m)$, be elements in $L$ which form a basis of $L$ by adding some basis-elements of $L_{\sigma}$. Consider the following lattices:

$$
\begin{aligned}
& L^{\prime}=\sum_{j=1}^{m} \mathbb{Z} \delta_{j}+\sum_{k=1}^{n-m} \mathbb{Z} \rho_{k}, \\
& D^{\prime}=\beta^{-1}\left(L^{\prime}\right) .
\end{aligned}
$$

Then the following sequence is exact:

$$
0 \rightarrow \mathbf{n} \stackrel{\iota}{\rightarrow} D^{\prime} \stackrel{\beta}{\rightarrow} L^{\prime} \rightarrow 0
$$

Through the homomorphism $\beta, D / D^{\prime}$ is isomorphic to $L / L^{\prime}$. Denote the group

$$
G_{x}:=D / D^{\prime} \simeq L / L^{\prime} .
$$

One has an exact sequence of tori :

$$
0 \rightarrow \mathbb{T}(\mathbf{n}) \rightarrow \mathbb{T}\left(D^{\prime}\right) \rightarrow \mathbb{T}\left(L^{\prime}\right) \rightarrow 0,
$$

and $G_{x}$ is imbedded into $\mathbb{T}\left(D^{\prime}\right)$ and $\mathbb{T}\left(L^{\prime}\right)$ compatibly with the above epimorphism. Denote

$$
U_{\sigma}^{\prime}=\operatorname{Spec} \mathbf{C}\left[\check{\sigma} \cap L^{\prime *}\right], V_{\tilde{\sigma}}^{\prime}=\operatorname{Spec} \mathbf{C}\left[\check{\widetilde{\sigma}} \cap D^{\prime *}\right] .
$$

Then $\beta$ induces an equivariant morphism (with respective to $\mathbf{T}\left(D^{\prime}\right)$ and $\mathbf{T}\left(L^{\prime}\right)$ ),

$$
\pi_{\sigma}^{\prime}: V_{\tilde{\sigma}}^{\prime} \rightarrow U_{\sigma}^{\prime}
$$


$\pi_{\sigma}^{\prime}$ defines a principle $\mathbb{T}(\mathbf{n})$-bundle on which the group $G_{x}$ acts as a bundle morphism through $(10)$. It is easy to see that $\pi_{\sigma}^{\prime}$ is equivalent to the trivial bundle,

$$
\text { pr: } U_{\sigma}^{\prime} \times \mathbb{T}(\mathbf{m}) \rightarrow U_{\sigma}^{\prime}
$$

Since the $G_{x}$-quotient of $\pi_{\sigma}^{\prime}$ gives the fibration (9), therefore the conditions of Defintion 1 for the fibration (7) are satisfied.

\section{\$3. Rational Picard Groups of Toric Varieties}

Let $\mathbb{P}_{(\Sigma, L)}$ be the same as in the previous section. We are going to describe the rational Picard group of $\mathbb{P}_{(\Sigma, L)}$ in terms of orbifold line bundles. Since

$$
H^{q}\left(\mathbb{P}_{(\Sigma, L)}, \mathscr{O}\right)=0, \text { for } q \geq 1
$$

we have a natural identification:

$$
\operatorname{Pic}\left(\mathbb{P}_{(\Sigma, L)}\right)_{\mathbb{K}} \simeq H^{2}\left(\mathbb{P}_{(\Sigma, L)}, \mathbb{K}\right), \quad \mathbb{R}=\mathbb{Q}, \mathbb{R}, \mathbb{C},
$$

by assigning to a line bundle its Chern class. It is known that the rational cohomology ring of $\mathbb{P}_{(\Sigma, L)}$ is the $\mathbb{Q}$-algebra generated by the classes of toric divisors [7] [10] [16]. Hence we have the following result, (see [16] Corollary 2.5.):

Lemma 3. Pic $\left(\mathbb{P}_{(\Sigma, L)}\right)_{\mathbb{Q}}$ is the $\mathbb{Q}$-space generated by the "line bundles" associated to the toric divisors in $\mathbb{P}_{(\Sigma, L)}$.

(Here a non-zero multiple of a toric divisor $D$ is a Cartier divisor, so one can talk about its "line bundle" in the rational Picard group of $\left.\mathbb{P}_{(\Sigma, L)}\right)$.

By Lemma 1 and 2, there associates an orbifold line bundle $\mathscr{O}(\chi)$ over $\mathbb{P}_{(\Sigma, L)}$ from the $\mathbb{T}(\mathbb{B})$-orbifold bundle $\mathbb{C}_{(\Sigma, L)}$ for a character $\chi$ of $\mathbb{T}(\mathbb{R})$. Hence we have the homomorphism

$$
\mathbb{m}_{\mathbf{Q}}^{*}\left(=\mathbb{n}\left(\sum, L\right)_{\mathbb{Q}}^{*}\right) \rightarrow \operatorname{Pic}\left(\mathbb{P}_{(\Sigma, L)}\right)_{\mathbb{Q}},
$$

which sends an element $\chi$ of $\mathbb{m}^{*}$ to $\mathscr{O}(\chi)$. Define the group

$$
G\left(\sum, L\right):=\left\{\varphi: \mathbb{C}_{(\Sigma, L)} \rightarrow \mathbb{C}_{(\Sigma, L)}, \mathbb{T}(\mathbb{R}) \text {-equivariant biregular morphism }\right\}
$$


Note that each element in $G(\Sigma, L)$ can always be extended to an automorphism of $\overline{\mathbf{C}_{(\Sigma, L)}}$. It has been shown in [6] that $G\left(\sum, L\right)$ is an affine algebraic group, consisting of all the $\mathbf{T}(\mathbf{n})$-biregular morphism of $\overline{\mathbf{C}_{(\Sigma, L)}}$. The $G\left(\sum, L\right)$-action on $\mathbf{C}_{(\Sigma, L)}$ induces a bundle morphism:

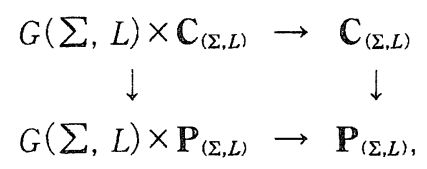

hence a $G\left(\sum, L\right)$-action on $\mathscr{O}(\chi)$. Therefore we obtain a representation of $G\left(\sum, L\right)$ on the vector space $H^{0}\left(\mathbf{P}_{(\Sigma, L)}, \mathscr{O}(\chi)\right)$.

Theorem 1. For a $n$-dimensional lattice $L$ and a complete fan $\sum$ in $L_{\mathbf{R}}$, let $d$ be the number of irreducible toric divisors in $\mathbb{P}_{(\Sigma, L)}$ and $\mathbf{n}$ be the $(d-n)$ dimensional lattice defined by (2). Then $\operatorname{Pic}\left(\mathbb{P}_{(\Sigma, L)}\right)_{\mathbb{Q}}$ is a $(d-n)$-dimensional vector space generated by toric divisors, and the homomorphism (12) induces a natural identification:

$$
\operatorname{Pic}\left(\mathbb{P}_{(\Sigma, L)}\right)_{\mathbb{Q}}=\mathbf{n}_{\mathbf{Q}}^{*}
$$

under which a character $\chi$ in $\mathbf{n}^{*}$ corresponds to the orbifold line bundle $\mathscr{O}(\chi)$ over $\mathbb{P}_{(\Sigma, L)}$. In particular, the "line bundle" associated to the linear equivalent class of toric divisor $e_{\delta}$ in $\mathbb{P}_{(\Sigma, L)}$ is given by $c^{*}\left(e_{\delta}^{*}\right)$ under the above identification.

Proof. We are going to show the homomorphism (12) is bijective. The coordinates of $\mathbb{C}_{(\Sigma, L)}$ are determined by the basis $\left\{e_{\delta}^{*}\right\}_{\delta \in \Sigma^{1}}$ of $D^{*}$, and the function of $\mathbf{C}_{(\Sigma, L)}$ for $e_{\delta}^{*}$ is a global section of $\mathscr{O}\left(\epsilon^{*}\left(e_{\delta}^{*}\right)\right)$ with its zeros at the toric divisor $e_{\delta}$. Hence the surjectivity of (12) follows from Lemma 3. Assume $\chi$ is a character of $\mathbb{T}(\mathbf{n})$ such that $\mathscr{O}\left(\chi^{N}\right)$ is the trivial line bundle over $\mathbb{P}_{(\Sigma, L)}$ for some positive integer $N$. There exists a never-vanishing holomorphic function $f$ on $\mathbb{C}_{(\Sigma, L)}$, which extends to $\overline{\mathbb{C}_{(\Sigma, L)}}$ by Hartogs' theorem, with

$$
f(z t)=\chi^{N}(t) f(z), \text { for } t \in \mathbb{T}(\mathbf{n}), z \in \overline{\mathbf{C}_{(\Sigma, L)}}
$$

This implies

$$
\chi^{N}(t) f(0)=f(0) \neq 0, \quad \text { for } t \in \mathbb{T}(\mathbf{n})
$$

here 0 is the 0 -dimensional $\mathbf{T}(D)$-orbit in $\overline{\mathbf{C}_{(\Sigma, L)}}$. Therefore $\chi^{N}$ is the trivial character, and $\chi=0 \in \mathbf{n}_{\mathbf{Q}}^{*}$. This shows the injectivity of (12). 
Remark. For the rest of this paper, we shall use the additive operation, instead of the multiplicative one, for the character group of $\mathbb{R}(\mathbb{R})$ which will be identified with $\mathbb{m}^{*}$. Then the canonical sheaf of $\mathbb{P}_{(\Sigma, L)}$ is given by the element $\iota^{*}(\kappa)$ in $\mathbb{R}^{*}$, where

$$
\kappa:=-\sum_{\delta \in \Sigma^{1}} e_{\delta}^{*} \in D_{0}^{*} \subset D_{\mathbb{Q}}^{*}
$$

e.g. for the ordinary projective $n$-space $\mathbb{P}^{n}$, the $e_{\delta}^{* \text { s }} \mathrm{s}$ form the $(n+1)$ homogeneous coordinates $x_{1}, \ldots, x_{n+1}$ and $\iota^{*}(\kappa)$ is equal to the line bundle associated to $-\sum_{i=1}^{n+1} D_{i}$ where $D_{i}$ the divisor of $\mathbb{P}^{n}$ defined by $x_{i}=0$.

Now we describe some properties related to the ampleness of $\mathscr{O}\left(\iota^{*} \rho\right)$ for $\rho$ $\in D^{*}$. For a $n$-dimensional lattice $L$, define the $(n+1)$-dimensional lattice,

$$
\bar{L}=L \otimes \mathbb{Z}
$$

and the element,

$$
q_{L}:=(0,1) \in \bar{L}
$$

By the natural inclusion and projection, one has the splitting exact sequence:

$$
0 \rightarrow \mathbb{Z} \rightarrow \bar{L} \rightarrow L \rightarrow 0
$$

It corresponds to the exact sequence of tori:

$$
0 \rightarrow \mathbb{C}^{*} \rightarrow \mathbb{T}(\bar{L}) \rightarrow \mathbb{T}(L) \rightarrow 0
$$

whose characters are given by the dual sequence of (14):

$$
0 \rightarrow L^{*} \rightarrow \overline{L^{*}} \rightarrow \mathbb{Z} \rightarrow 0
$$

For $\rho$ in $D^{*}$, we shall denote

$$
\rho_{\delta}:=\rho\left(e_{\delta}\right) \in \mathbb{Z}, \quad \text { for } \delta \in \Sigma^{1}
$$

The piecewise linear functional $f_{\rho}$ on $L_{\mathbb{R}}\left(=\mid \sum_{i}^{\prime}\right)$,

$$
f_{\rho}: L_{\mathbb{R}} \rightarrow \mathbb{R}
$$


is defined by $f_{\rho}(\delta)=\rho_{\delta}$ for $\delta \in \Sigma^{1}$, and linear on each cone in $\sum$. Consider the graph of $f_{\rho}$ :

$$
L_{\mathbf{R}} \rightarrow \bar{L}_{\mathbf{R}}\left(=L_{\mathbf{R}} \oplus \mathbf{R}\right), \quad x \mapsto\left(x, f_{\rho}(x)\right)
$$

The fan $\sum$ of $L_{\mathbb{R}}$ gives rise to a simplicial cone decomposition of the above graph, denoted by

$$
\sum(\rho)=\left\{\gamma(\sigma) \mid \sigma \in \sum\right\}, \quad \gamma(\sigma):=\text { the graph of } f_{\rho} \text { over the region } \sigma .
$$

Then $\sum(\rho)$ is a rational fan in $\bar{L}_{\mathbf{R}}$ with its support $\left|\sum(\rho)\right|$ equal to the graph of $f_{\rho}$. Let $E_{\rho}^{*}$ be the $\mathrm{T}(\bar{L})$-toric variety associated to $\sum(\rho)$, hence it is acted by $\mathbb{C}^{*}$ via the sequence (15). One has the equivariant morphism from $\mathbb{T}(\bar{L})$ -variety $E_{\rho}^{*}$ onto the $\mathbb{T}(L)$-variety $\mathbb{P}_{(\Sigma, L)}$.

$$
\pi_{\rho}: E_{\rho}^{*} \rightarrow \mathbb{P}_{(\Sigma, L)}
$$

extending the projection of $\mathbb{T}(\bar{L})$ to $\mathbb{T}(L)$. It is not hard to see that the above fibration $\pi_{\rho}$ is a principal orbifold $\mathbb{C}^{*}$-bundle. We are going to construct a partial compactification of $E_{\rho}^{*}$ along fibers of $\pi_{\rho}$ as follows. Let $\mathscr{C}_{\rho}$ be the epigraph of $f_{\rho}$, i.e.

$$
\mathscr{C}_{\rho}:=\left\{(x, \alpha) \in \bar{L}_{\mathbf{R}} \mid \alpha \geq f_{\rho}(x), \quad x \in L_{\mathbf{R}}\right\}
$$

$\mathscr{C}_{\rho}$ is a $(n+1)$-dimensional cone whose interior contains the element $q_{L}$. A simplicial cone decomposition of $\mathscr{C}_{\rho}$ is obtained by starring $\sum(\rho)$ at $q_{L}$ :

$$
\sum(\rho) * q_{L}=\bigcup_{\gamma(\sigma) \in \Sigma(\rho)}\left\{\text { faces of }\left(\gamma(\sigma)+\mathbb{R}_{\geq 0} q_{L}\right)\right\}
$$

which is a fan in $\bar{L}_{\mathbf{R}}$ containing $\sum(\rho)$ with $\left|\sum(\rho) * q_{L}\right|=\mathscr{C}_{\rho}$. Let $E_{\rho}$ be the $\mathrm{T}(\bar{L})$-variety associated to the fan $\sum(\rho) * q_{L}$. The projection,

$$
\sum(\rho) * q_{L} \rightarrow \sum
$$

gives rise to an equivariant morphism from $E_{\rho}$ onto $\mathbb{P}_{(\Sigma, L)}$. By the commutative diagram 


$$
\begin{array}{ccc}
E_{\rho}^{*} & \subset & E_{\rho} \\
\downarrow \pi_{p} & & \downarrow \pi_{p} \\
\mathbb{P}_{(\Sigma, L)} & = & \mathbb{P}_{(\Sigma, L)},
\end{array}
$$

$E_{\rho}-E_{\rho}^{*}$ isomorphic to $\mathbb{P}_{(\Sigma, L)}$ under the map $\pi_{\rho}$, and $E_{\rho}$ is a partial compactification of $E_{\rho}^{*}$. On the other hand, the homomorphism,

$$
p_{\rho}: D \rightarrow \vec{L}, x \mapsto(\beta(x), \rho(x))
$$

induces a tori-morphism

$$
\mathbb{T}(D) \rightarrow \mathbb{T}(\bar{L})
$$

One has the commutative diagram of exact sequences:

$$
\begin{aligned}
& 0 \rightarrow \mathbb{n} \stackrel{\iota}{\rightarrow} D \stackrel{\beta}{\rightarrow} L \rightarrow 0 \\
& \downarrow i \rho \quad \downarrow p_{\rho} \quad \| \\
& 0 \rightarrow \mathbb{Z} \rightarrow \bar{L} \rightarrow L \rightarrow 0,
\end{aligned}
$$

and its dual diagram:

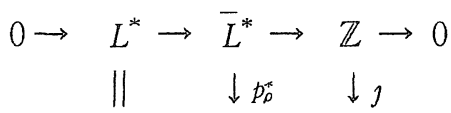

$$
\begin{aligned}
& 0 \rightarrow L^{*} \stackrel{\beta^{*}}{\rightarrow} D^{*} \stackrel{\iota^{+}}{\rightarrow} \mathbb{R}^{*} \rightarrow 0 \text {, }
\end{aligned}
$$

where $\jmath$ is the linear map sending 1 to $\iota^{*} \rho$, and $p_{o}^{*}$ has the expression

$$
p_{\rho}^{*}: \bar{L}^{*} \rightarrow D^{*},\left(l^{\prime}, a\right) \mapsto \beta^{*}\left(l^{\prime}\right)+a \rho \quad \text { for } a \in \mathbb{R}, l^{\prime} \in L_{\mathbb{R}}^{*}
$$

For $\sigma \in \sum, \gamma(\sigma)$ is generated by $\left\{p_{\rho}\left(e_{\delta}\right) \mid \delta \in \sigma \cap \Sigma^{1}\right\}$, and the linear map,

$$
p_{\rho}: D_{\mathbb{R}} \rightarrow \bar{L}_{\mathbb{R}}
$$

sends the simplicial cone $\widetilde{\sigma}$ of $(6)$ in $D_{\mathbf{R}}$ to $\gamma(\sigma)$. Hence we have the expression

$$
\sum(\rho)=\left\{p_{\rho}(\widetilde{\sigma}) \mid \sigma \in \sum\right\},
$$


from which one obtains the induced "bundle" morphism:

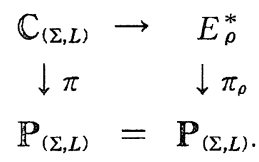

With the character $\iota^{*} \rho$ of $\mathbf{T}(\mathbf{n})$ on $\mathbf{C}_{(\Sigma, L)}$ and the identity character of $\mathbb{C}^{*}$ on $E_{\rho}^{*}$ in Definition 2, one has the commutative diagram:

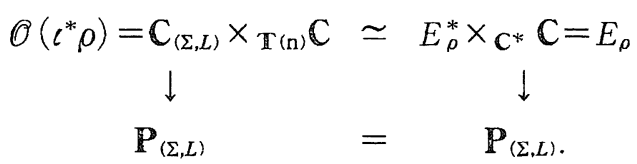

In this manner, $E_{\rho}^{*}$ is identified with the complement of the zero section of $\mathscr{O}(-$ $\left.\imath^{*} \rho\right)$ over $\mathbf{P}_{(\Sigma, L)}$, and (19) is equivalent to the diagram :

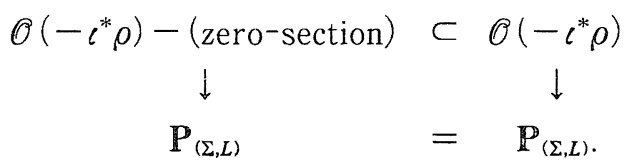

From now on, we consider only those $\mathscr{O}\left(\iota^{*} \rho\right)$ having non-trivial sections. Without loss of generality we shall always make the following assumption on $\rho$ for the rest of this paper:

$$
\rho=\sum_{\delta \in \Sigma^{1}} \rho_{\delta} e_{\delta}^{*} \in D^{*}-\{0\}, \quad \rho_{\delta} \in \mathbb{Z}_{\geq 0} \text { for all } \delta,
$$

or equivalently, $f_{\rho}$ is a non-negative and non-trivial function on $L_{\mathbf{R}}$. Note that the cone $\mathscr{C}_{\rho}$ in $\bar{L}_{\mathbb{R}}$ is non-convex in general; while the $p_{\rho}$-image of first quadrant cone $\Omega$ of $D_{\mathbf{R}}, p_{\rho}(\Omega)$, is always a convex polyhedral cone, which is characterized as the convex cone in $\bar{L}_{\mathbf{R}}$ generated by $\left(\delta, \rho_{\delta}\right), \delta \in \Sigma^{1}$. Since $\rho$ is not trivial, $p_{\rho}(\Omega)$ is a $(n+1)$-dimensional cone containing $q_{L}$ and the relation

$$
\mathscr{C}_{\rho} \subseteq p_{\rho}(\Omega)
$$

holds. The equality of the above sets is related to the convexity of $\rho$ in the context of ample toric line bundles [7] [12] [15]. As the generating set $\sigma \cap$

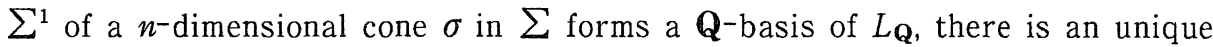
$\mathbf{Q}$-linear functional $l_{\sigma}$ of $L_{\mathbf{Q}}$ with the property: 


$$
l_{\sigma}(\delta)=\rho_{\delta}, \quad \text { for } \delta \in \sigma \cap \sum^{1}
$$

The following definition is given in [16] [19]:

Definition 3. For an element $\rho$ in (23), we say

(i) $\rho$ is convex if the functional $f_{\rho}$ on $L_{\mathbb{R}}$ is a convex function, i.e.

$$
\begin{aligned}
& l_{\sigma}(x) \leq f_{\rho}(x), \quad \text { for } \sigma \in \sum^{(n)}, x \in L_{\mathbb{R}}, \\
& \Leftrightarrow f_{\rho}(x+y) \leq f_{\rho}(x)+f_{\rho}(y), \quad \text { for } x, y \in L_{\mathbb{R}} \text {. }
\end{aligned}
$$

(ii) $\rho$ is strictly convex if $f_{\rho}$ is a strictly convex function, i.e. for $\sigma \in \sum^{(n)}$ and $x \in L_{\mathbb{R}}$,

$$
l_{\sigma}(x) \leq f_{\rho}(x), \quad \text { and } "=" \text { holds iff } x \in \sigma .
$$

Proposition $\mathbb{1}$. The following conditions are equivalent:

(i) $\rho$ is convex if and only if $\mathscr{C}_{\rho}$ is a convex cone. In this situation, the following equality holds :

$$
\mathscr{C}_{\rho}=p_{\rho}(\Omega)
$$

(ii) $\rho$ is strictly convex if and only if $\mathscr{C}_{\rho}$ is a convex cone in $\bar{L}_{\mathbb{R}}$ having all the proper faces to be simplicial and with $\left\{\left(\delta, \rho_{\delta}\right) \mid \delta \in \Sigma^{1}\right\}$ as the fundamental generators, (i.e. irredundant ones). In this situation, the cone $\mathscr{C}_{\rho}$ is strongly convex, i.e. $\mathscr{C}_{\rho} \cap\left(-\mathscr{C}_{\rho}\right)=\{0\}$.

Proof. Since the elements $\left(\delta, \rho_{\delta}\right)$ of $\mathscr{C}_{\rho}$ generate the convex cone $p_{\rho}(\Omega)$, the convex condition for $\mathscr{C}_{\rho}$ is equivalent to the equality of $\mathscr{C}_{\rho}$ and $p_{\rho}(\Omega)$. For $\sigma \in$ $\Sigma^{(n)}, \gamma(\sigma)$ is a $n$-dimensional cone in $\bar{L}_{\mathbb{R}}$ generated by elements $\left(\delta, \rho_{\delta}\right)$ for $\delta \in$ $\sigma \cap \Sigma^{1}$. The graph of $l_{\sigma}$ in $\bar{L}_{\mathbb{R}}$ coincides with $\gamma(\sigma)$ over the region $\sigma$. Therefore the condition of $\gamma(\sigma) \subseteq \partial p_{\rho}(\Omega)$ becomes $\rho(x) \geq l_{\sigma}(x)$ for $x \in \Omega$, which is equivalent to

$$
f_{\rho}(\delta)\left(=\rho_{\delta}\right) \geq l_{\sigma}(\delta) \text { for } \delta \in \Sigma^{1}
$$

Therefore we obtain (i). It is not hard to see that the strictly convexity of $\rho$ is equivalent to the irredundant condition of the generators $\left(\delta, \rho_{\delta}\right)$ 's together with the simplical proper face-property of $p_{\rho}(\Omega)$. In this situation, we have $\mathscr{C}_{\rho}=$ $p_{o}(\Omega)$. If $p_{\rho}(\Omega)$ contains a linear space generated by a non-trivial element $v$ in $\bar{L}_{\mathbb{R}}$, write 


$$
v=\left(x_{v}, \alpha\right), x_{v} \in L_{\mathbf{R}}, \quad \alpha \in \mathbf{R} .
$$

$x_{v}$ belongs to some $n$-dimensional cone $\sigma$ of $\sum$. Since $p_{\rho}(\Omega)$ is contained in the "upper" half-space of $\bar{L}_{\mathbf{R}}, \alpha$ is equal to 0 , hence $\pm v \in \partial p_{\rho}(\Omega)$. This implies $l_{\sigma}\left(x_{v}\right)=0$. By the strictly convex condition of $\rho$ and $-x_{v} \notin \sigma, f_{\rho}\left(-x_{v}\right)>l_{\sigma}\left(-x_{v}\right)$ $=0$, which contradicts $-v\left(=\left(-x_{v}, 0\right)\right) \in p_{\rho}(\Omega)$. Therefore $\mathscr{C}_{\rho}$ is strongly convex.

As a consequence of Proposition 1, the ampleness for a multiple of anti-canonical divisor of $\mathbf{P}_{(\Sigma, L)}$ is now determined by the polytope $\Delta(L)$ of $(1)$ :

Corollary. Let $\kappa$ be the element (13) and $r$ a negative rational number such that $r \kappa \in D^{*}$. Then

$$
\begin{array}{ll}
r \kappa: \text { convex } & \Leftrightarrow \Delta(L) \text { : convex; } \\
r \kappa: \text { strictly convex } & \Leftrightarrow \Delta(L) \text { : convex cone with simplical proper faces and } \\
& \text { having }\{\delta\}_{\delta \in \Sigma^{1}} \text { as a set of minimal gener. } \\
& \text { ators. }
\end{array}
$$

For a convex element $\rho, \mathscr{C}_{\rho}$ is a $(n+1)$-dimensional convex cone in the upper half-space of $\bar{L}_{\mathbf{R}}$. Let $\mathrm{v}_{\rho}$ be the maximal linear subspace of $L_{\mathbf{R}}$ contained in $\mathscr{C}_{\rho}$, and $\mathrm{v}_{\rho}^{\perp}$ be its annihilating subspace in $L_{\mathbf{R}}^{*}$. Then the dual cone $\mathscr{C}_{\rho}^{*}$ is a convex cone in $\mathrm{v}_{\rho}^{\perp}+\mathbb{R} q_{L^{*}}$ with $q_{L^{*}} \in \operatorname{Int}\left(\mathscr{C}_{\rho}^{*}\right)$ :

$$
q_{L^{*}} \in \mathscr{C}_{\rho}^{*} \subseteq \mathrm{v}_{\rho}^{\perp}+\mathbf{R} q_{L^{*}} \subseteq \bar{L}_{\mathbf{R}}
$$

The relation between $\mathscr{C}_{\rho}^{*}$ and the dual first quardrant cone $\Omega^{*}\left(=\sum_{\delta \in \Sigma^{1}} \mathbf{R}_{\geq 0} e_{\delta}^{*}\right)$ of $D_{\mathbf{R}}^{*}$ is given by $p_{\rho}^{*}$ :

$$
p_{\rho}^{*}: \mathscr{C}_{\rho}^{*} \simeq \Omega^{*} \bigcap p_{\rho}^{*}\left(\overline{L_{\mathbf{R}}^{*}}\right) .
$$

Since the boundary of $\mathscr{C}_{\rho}$ is the graph of $f_{\rho}$, every 1 -face of $\mathscr{C}_{\rho}^{*}$ must intersect the $\left(q_{L}=1\right)$-hyperplane at one point. $\mathscr{C}_{\rho}^{*}$ is determined by its cross section with the $\left(q_{L}=1\right)$-hyperplane. Let $\Delta\left(L^{*}\right)_{\rho}$ be the projection of this cross section to $L_{\mathbf{R}}$ :

$$
\Delta\left(L^{*}\right)_{\rho}:=\left\{x \in L_{\mathbf{R}}^{*} \mid(x, 1) \in \mathscr{C}_{\rho}^{*}\right\}
$$


Then $\Delta\left(L^{*}\right)_{\rho}$ is a compact convex polytope in $\mathrm{v}_{\rho}^{\perp}$, hence in $L_{\mathbb{R}}^{*}$. In the next section we shall show that integral elements in $\Delta\left(L^{*}\right)_{\rho}$ determine the sections of $\mathscr{O}\left(\iota^{*} \rho\right)$ over $\mathbb{P}_{(\Sigma, L)}$.

\section{\$4. Quasi-smooth Hypersurfaces in Torie Varieties}

First let us recall the notion of quasi-smoothness of a hypersurface in a toric variety introduced by Batyrev and Cox in [5]. A hypersurface $X$ in $\mathbb{P}_{(\Sigma, L)}$ is called to be quasi-smooth if $\pi_{0}^{*}(X)$ is non-singular in $\mathbb{C}_{\left(\Sigma, L_{0}\right)}$ where $\pi_{0}$ is the morphism in (8). This concept is equivalent to "simplicially toroidal pairs" by Danilov [9]. In this section we are going to discuss some basic properties of quasi-smooth hypersurfaces defined by sections of an orbifold line bundle over $\mathbb{P}_{(\Sigma, L)}$. Let $\rho$ be a convex element of $D^{*}$ with the cone $\mathscr{C}_{\rho}$ in $\bar{L}_{\mathbb{R}}$, its dual convex cone $\mathscr{C}_{\rho}^{*}$ in $\overline{L^{*}}$, and the convex polytope $\Delta\left(L^{*}\right)_{\rho}$ in $L_{\mathbb{R}}^{*}$.

Proposition 2。 The vector space $\Gamma\left(\mathbb{P}_{(\Sigma, L)}, \mathscr{O}\left(\iota^{*} \rho\right)\right)$ has a basis consisting of elements $z \in \overline{L^{*}} \cap \mathscr{C}_{\rho}^{*}$ with $\left\langle q_{L}, z\right\rangle=1$, which are in one-to-one correspondence with elements in $L^{*} \cap \Delta\left(L^{*}\right)_{\rho}$, i.e.

$$
\begin{aligned}
\Gamma\left(\mathbb{P}_{(\Sigma, L)}, \mathscr{O}\left(\iota^{*} \rho\right)\right) & \simeq \oplus\left\{\mathbb{C}_{z} \mid z \in \overline{L^{*}} \cap \mathscr{C}_{\rho}^{*},\left\langle q_{L}, z\right\rangle=1\right\} \\
& \simeq \oplus\left\{\mathbb{C} x \mid x \in L^{*} \cap \Delta\left(L^{*}\right)_{\rho}\right\}
\end{aligned}
$$

Proof. Consider the fibration $E_{\rho}$ over $\mathbb{P}_{(\Sigma, L)}$ in (19). It is isomorphic to the orbifold line bundle $\mathscr{O}\left(-\iota^{*} \rho\right)$ over $\mathbb{P}_{(\Sigma, L)}$ with the scalar multiplication corresponding to the action of one-parameter subgroup $q_{L}$ on $E_{\rho}$. Hence the sections in $\Gamma\left(\mathbb{P}_{(\Sigma, L)}, \mathscr{O}\left(\iota^{*} \rho\right)\right)$ can be regarded as regular functions of $E_{\rho}$. linear with respect to the $\mathbb{C}^{*}$-action induced by $q_{L}$. By $|\widetilde{\Sigma}(\rho)|=\mathscr{C}_{\rho}, \overline{L^{*}} \cap \mathscr{C}_{\rho}^{*}$ determines the regular functions of $E_{\rho}$. Hence a basis of $\Gamma\left(\mathbb{P}_{(\Sigma, L)}, \mathscr{O}\left(\iota^{*} \rho\right)\right)$ consists of the elements in $\overline{L^{*}} \cap \mathscr{C}_{\rho}^{*}$ whose $q_{L}$-values equal to 1 . It is easy to see that they are in one-to-one correspondence with elements in $L^{*} \cap \Delta\left(L^{*}\right)_{\rho}$.

By the Proposition 2 , a section $s$ in $\Gamma\left(\mathbb{P}_{(\Sigma, L)}, \mathscr{O}\left(\iota^{*} \rho\right)\right)$ has the following expression :

$$
s=\sum_{x \in L^{*} \cap \Delta\left(L^{*}\right) \rho} \alpha_{x} x, \quad \text { or equivalently } s=\sum_{z \in \overline{L^{*}} \cap \mathscr{C}_{p}^{*},<q_{L}, z>=1} \alpha_{z} z
$$

One can consider $s$ as a function on the $\mathbb{T}(\bar{L})$-variety $E_{\rho}\left(\simeq \mathscr{O}\left(-\iota^{*} \rho\right)\right)$. The 
Newton polygon of $s$ is defined to be the convex hull in $L_{\mathbf{R}}^{*}$ spanned by $x$ 's with $\alpha_{x} \neq 0$, hence is contained in $\Delta\left(L^{*}\right)_{\rho}$. Let $X$ be the hypersurface of $\mathbf{P}_{(\Sigma, L)}$ defined by the zeros of $s$. We are going to describe the local defining equation of $X$. For $\sigma \in \Sigma^{(n)}$, we have the affine open subset $U_{\sigma}$ of $\mathbf{P}_{(\Sigma, L)}$ :

$$
U_{\sigma}=\operatorname{Spec} \mathbf{C}\left[\check{\sigma} \cap L^{*}\right] \subset \mathbb{P}_{(\Sigma, L)}
$$

Denote

$$
\left\{\delta_{i}\right\}_{i=1}^{n}=\sum^{1} \cap \sigma
$$

and let $I_{\sigma}$ be the finite subgroup of $\mathbf{C}^{n *}$ defined by the image of $L$ under the following homomorphism :

$$
\sum_{\imath=1}^{n} \mathbb{R} \delta_{\imath} \rightarrow \mathbb{C}^{n \psi}, \quad \sum_{\imath=1}^{n} x_{i} \delta_{\imath} \mapsto\left(e^{2 \pi \imath x_{1}}, \ldots, e^{2 \pi \imath \cdot x_{n}}\right)
$$

Then $I_{\sigma}$ is isomorphic to $L / \sum_{t=1}^{n} \mathbb{Z} \delta_{i}$, and $U_{\sigma}$ is realized as an orbifold through the projection :

$$
\phi: \mathbb{C}^{n} \rightarrow L_{\sigma}=\mathbb{C}^{n} / I_{\sigma}
$$

where the dual basis of $\left\{\delta_{i}\right\}_{i=1}^{n}$ gives rise to the coordinates $\left(t_{1}, \ldots, t_{n}\right)$ of $\mathbb{C}^{n}$. By Proposition 3. 5 of [5], quasi-smooth hypersurfaces have the following local description. $X \cap U_{\sigma}$ is quasi-smooth if $\psi^{*}\left(X \cap U_{\sigma}\right)$ is a smooth hypersurface in $\mathbb{C}^{n}$, and we call $X$ to be quasi-smooth if $X \cap U_{\sigma}^{r}$ is quasi-smooth for all $\sigma \in$ $\sum^{(n)}$. Let

$$
\left\{\begin{array}{l}
p_{i}=\left(\delta_{i}, \rho_{\delta_{1}}\right), \text { for } 1 \leq i \leq n, \\
p_{n+1}=q_{L}
\end{array}\right.
$$

All the $p_{i}$ 's are primitive elements in $\bar{L}$ and they generate a $(n+1)$-simplicial cone in $\Sigma(\rho)$. The first $n$ elements $p_{1}, \ldots, p_{n}$, lie in an unique $n$-dimensional face of $\mathscr{C}_{\rho}$, annihilated by a vertex $z(\sigma)$ of the polytope $\mathscr{C}_{\rho}^{*} \cap\left(q_{L}=1\right)$. Note that $z(\sigma)$ may not be an element in $\overline{L^{*}}$ unless $\Delta\left(L^{*}\right)_{\rho}$ is the Newton polygon of $s$. The dual elements $p_{j}^{*}, 1 \leq j \leq(n+1)$, are in $\overline{L^{*}}$, with

$$
\left\langle p_{i}, p_{j}^{*}\right\rangle=\delta_{i j}, 1 \leq i, j \leq(n+1) .
$$


Hence

$$
p_{1}^{*}, \ldots, p_{n}^{*} \in L_{\mathbb{Q}}^{*}, \quad p_{n+1}^{*}=z(\sigma)
$$

and $\left\{p_{i}^{*}\right\}_{i=1}^{n}$ is the dual basis of $\left\{\delta_{i}\right\}_{\imath=1}^{n}$. By

$$
\mathscr{C}_{\rho}^{*} \subset \sum_{j=1}^{n+1} \mathbb{R}_{\geq 0} p_{j}^{*}
$$

for $z \in \overline{L^{*}} \cap \mathscr{C}_{\rho}^{*}$ with $\left\langle q_{L}, z\right\rangle=1$, one has

$$
y_{\sigma}(z):=z-z(\sigma)=\sum_{i=1}^{n} k(z)_{i} p_{i}^{*}, \quad \text { with } k(z)_{i}=\left\langle p_{i}, z\right\rangle \in \mathbb{Z}_{\geq 0}
$$

Consider $y_{\sigma}(z)$ as a regular function of $U_{\sigma}$. Through (27), one has

$$
\phi^{*}\left(y_{\sigma}(z)\right)=\prod_{i=1}^{n} t_{\imath}^{k(z)_{\imath}}
$$

and we obtain the defining equation for $X \cap U_{\sigma}$,

$$
X \bigcap U_{\sigma}: \sum_{z \in \overline{L^{*}} \cap \mathscr{G}_{D,}^{*},\left\langle q_{L}, z>=1\right.} \alpha_{z} y_{\sigma}(z)=0
$$

The hypersurface $\phi^{*}\left(X \cap U_{\sigma}\right)$ in $\mathbb{C}^{n}$ is now defined by

$$
\eta_{\sigma}: \sum_{z \in \bar{L}^{*} \cap \mathscr{C}_{p,}^{*}<q_{L^{\prime}}, z>=1} \alpha_{z} \prod_{i=1}^{n} t_{i}^{k(z)_{i}}=0
$$

Now assume $\mathscr{C}_{\rho}$ is a strongly convex polyhedral cone in $L_{\mathbb{R}}$. One has the order reversing bijective map:

$$
\begin{aligned}
& \text { fface of } \left.\mathscr{C}_{\rho}\right\} \longleftrightarrow\left\{\text { face of } \mathscr{C}_{\rho}^{*}\right\} \\
& \tau \quad \leftrightarrow \quad \tau^{\prime}:=\mathscr{C}_{\rho}^{*} \cap \tau^{\perp}
\end{aligned}
$$

with $\operatorname{dim}(\tau)+\operatorname{dim}\left(\tau^{\prime}\right)=n . \tau^{\prime}$ is called the dual of $\tau$ in $\mathscr{C}_{\rho}^{*}$. The dual of a facet, (i.e. codimensional one face), of $\mathscr{C}_{\rho}$ is the 1 -face of $\mathscr{C}_{\rho}^{*}$ generated by $(v, 1)$ for some vertex $v$ of $\Delta\left(L^{*}\right)_{\rho}$, and we have 


$$
v \in L^{*} \Longleftrightarrow(v, 1) \in \overline{L^{*}}
$$

Lemma 4. Let $X$ be a hypersurface of $\mathbf{P}_{(\Sigma, L)}$ defined by zeros of a section $s \in$ $\Gamma\left(\mathbf{P}_{(\Sigma, L)}, \mathscr{O}\left(\iota^{*} \rho\right)\right)$. For a vertex $v$ of $\Delta\left(L^{*}\right)_{\rho}$, let $\tau$ be the facet of $\mathscr{C}_{\rho}$ dual to $(v, 1)$ $\in \mathscr{C}_{\rho}^{*}$. If the Newton polygon of $s$ contains $v$, then

$$
X \bigcap \bigcup_{r(\sigma) \subset \tau} \operatorname{orb}(\sigma)=\varnothing
$$

where orb $(\sigma)$ is the $\mathbb{T}(L)$-orbit in $\mathbb{P}_{(\Sigma, L)}$ and $\gamma(\sigma)$ is the cone (16) in the fan $\sum(\rho)$ associated to $\sigma$.

Proof. Regard $s$ as a function of $E_{\rho}^{*}\left(=\mathscr{O}\left(-\iota^{*} \rho\right)-(\right.$ zero-section $\left.)\right)$. For $t$ $\in \operatorname{Int}(\tau)$ and $x \in L^{*} \cap \Delta\left(L^{*}\right)_{\rho}$ with $\alpha_{x} \neq 0$ in (25), we have

$$
\langle t,(x, 1)\rangle \geq 0 \text {, and } "=" \text { holds iff } x=v \text {. }
$$

For $\gamma(\sigma) \subset \tau$ and an element $e$ of $E_{\rho}^{*}$ lying over $\operatorname{orb}(\sigma)$, we have

$$
s(e)=\alpha_{v} v(e) \neq 0
$$

Therefore the conclusion follows immediately.

Definition 4. Let $M$ be a lattice. A convex polytope in $M_{\mathbf{R}}$ is called integral (with respect to $M$ ) if all its vertices are in $M$.

The following result is obvious.

Lemma 5. The following conditions are equivalent:

(i) Newton polygon of $s$ is equal to $\Delta\left(L^{*}\right)_{\rho}$.

(ii) $\Delta\left(L^{*}\right)_{\rho}$ is an integral polytope in $L_{\mathbf{R}}^{*}$, and the coefficient $\alpha_{x}$ in (25) is non-zero for each vertex $x$ of $\Delta\left(L^{*}\right)_{\rho}$.

Remark. For an integral polytope $\Delta\left(L^{*}\right)_{\rho}$, a generic section $s$ of $\Gamma\left(\mathbf{P}_{(\Sigma, L)}\right.$, $\left.\mathfrak{O}\left(\iota^{*} \rho\right)\right)$ always satisfies the above lemma, and it defines a quasi-smooth hypersurface of $\mathbb{P}_{(\Sigma, L)}$.

For a strictly convex element $\rho, \mathscr{C}_{\rho}$ is a strongly convex polyhedral cone in $\vec{L}_{\mathbf{R}}$ by Proposition 1 , hence $\Delta\left(L^{*}\right)_{\rho}$ is a $n$-dimensional convex polytope in $L_{\mathbf{R}}^{*}$. Let $A_{\rho}$ be the affine $\mathbf{T}(\bar{L})$-variety associated to the cone $\mathscr{C}_{\rho}$ : 


$$
A_{\rho}=\operatorname{Spec} \mathbb{C}\left[\bar{L}^{*} \cap \mathscr{C}_{\rho}^{*}\right]
$$

Since $\left\{\left(\delta, \rho_{\delta}\right) \mid \delta \in \Sigma^{1}\right\}$ forms a system of fundamental generators of $\mathscr{C}_{\rho}, A_{\rho}$ is the $\mathbb{T}(\bar{L})$-variety obtained by adding one point (= the 0 -dimensional orbit) to $E_{\rho}^{*}$. In fact, $A_{\rho}$ is the affine variety obtained by blowing down the zero section of the orbifold line bundle $\mathfrak{O}\left(-\iota^{*} \rho\right)$ over $\mathbb{P}_{(\Sigma, L)}$ :

$$
E_{\rho}=\mathfrak{O}\left(-\iota^{*} \rho\right) \rightarrow A_{\rho}
$$

and the one-parameter subgroup $q_{L}$ gives the $\mathbb{C}^{*}$-action on $A_{\rho}$ :

$$
\mathbb{C}^{*} \times A_{\rho} \rightarrow A_{\rho},(\lambda, z) \mapsto \lambda \cdot z
$$

By Proposition 2, one has the identification:

$$
\Gamma\left(\mathbb{P}_{(\Sigma, L)}, \mathscr{O}\left(\iota^{*} \rho\right)\right)=\left\{f: A_{\rho} \rightarrow \mathbb{C} \mid f(\lambda \cdot z)=\lambda f(z) \text { for } \lambda \in \mathbb{C}^{*}, z \in A_{\rho}\right\}
$$

The affine coordinates of $A_{\rho}$ can be considered as the "homogeneous" coordinates of $\mathbb{P}_{(\Sigma, L)}$ for sections of $\mathscr{O}\left(\iota^{*} \rho\right)$. The Lefschetz-type theorem on the cohomology of $\mathbb{P}_{(\Sigma, L)}$ and its hypersurface was obtained by Danilov and Khovanskii [8]. The following form can be derived by a similar argument as in Theorem 2 of [1]. We repeat it here for the sake of completeness.

Proposition 3. Let $\rho$ be a strictly convex element in $D^{*}$, and $X$ be a quasi-smooth hypersurface of $\mathbb{P}_{(\Sigma, L)}$ defined by a section of $\mathscr{O}\left(\iota^{*} \rho\right)$. Then the homomorphism

$$
H^{i}\left(\mathbb{P}_{(\Sigma, L)}, \mathbb{C}\right) \rightarrow H^{i}(X, \mathbb{C})
$$

induced by inclusion is an isomorphism for $i<n-1$, and injective for $i=n-1$.

Proof. Let $U$ be the complement of $X$ in $\mathbb{P}_{(\Sigma, L)}$. The quasi-smooth property for $X$ and $U$ implies that $U$ is a rational homology manifold where Poincare duality holds for the cohomology (with complex coefficients throughout) :

$$
H^{\imath}\left(\mathbb{P}_{(\Sigma, L)}, X\right) \simeq H_{2 n-\imath}(U)
$$

Since $\mathscr{O}\left(\iota^{*} \rho\right)$ is an ample line bundle over $\mathbb{P}_{(\Sigma, L)}, U$ is an affine variety, therefore a Stein space. By the acyclic resolution of the constant sheaf $\mathbb{C}_{U}$ : 


$$
0 \rightarrow \underline{\mathrm{C}}_{U} \rightarrow \Omega_{\dot{U}}\left(=\left\{\Omega_{U}^{0} \stackrel{d}{\rightarrow} \Omega_{U}^{1} \stackrel{d}{\rightarrow} \cdots \stackrel{d}{\rightarrow} \Omega_{U}^{n} \rightarrow 0\right\}\right)
$$

one has

$$
H^{\prime}(U)=0 \text { for } j>n
$$

hence

$$
H^{2}\left(\mathbb{P}_{(\Sigma, L)}, X\right) \simeq H_{2 n-i}(U)=0, \text { for } \quad j \leq n
$$

By the exact cohomology sequence :

$$
\cdots \rightarrow H^{\imath}\left(\mathbb{P}_{(\Sigma, L)}, X\right) \rightarrow H^{\imath}\left(\mathbb{P}_{(\Sigma, L)}\right) \rightarrow H^{\imath}(X) \rightarrow H^{\imath+1}\left(\mathbb{P}_{(\Sigma, L)}, X\right) \rightarrow \cdots
$$

the conclusion follows immediately.

Remark. A similar statement can be found in Theorem 3.7 of [8]. The relation between Hodge groups of $X$ and $\mathbf{P}_{(\Sigma, L)}$ can be derived from the above proposition, for the argument see Appendix (I).

\section{§5. Examples}

In this section, some examples are given for the illustration of the results obtained in previous sections.

(I) The case for $n=1$. We have

$$
\begin{aligned}
L & =\mathbb{Z}, \\
\sum & =\left\{\mathbb{R}_{\geq 0}, \mathbb{R}_{\leq 0}, 0\right\}, \\
\sum^{1} & =\left\{\delta_{+}, \delta_{-}\right\}, \delta_{ \pm}= \pm 1, \\
\mathbf{P}_{(\Sigma, L)} & =\mathbb{P}^{1},
\end{aligned}
$$

hence

$$
D=\mathbf{Z}^{2}\left(=\mathbb{Z}_{e_{\delta_{+}}}+\mathbf{Z}_{e_{-}}\right) .
$$

The map $\beta$ is given by

$$
\beta: D \rightarrow L,\left(m_{1}, m_{2}\right) \mapsto m_{1}-m_{2},
$$


with the kernel

$$
\mathbb{n}=\mathbb{Z}(1,1) .
$$

The $\mathbb{C}^{*}$-bundle $(7)$ is the well-known Hopf fibration:

For a given

$$
\pi: \mathbb{C}^{2}-\{0\} \rightarrow \mathbb{P}^{1}
$$

$$
\rho=(k, l) \in D^{*}-\{0\}, k, l \in \mathbb{Z}_{\geq 0}
$$

one has

$$
\mathscr{C}_{\rho}=\mathbb{R}_{\geq 0}(1, k)+\mathbb{R}_{\geq 0}(-1, l),
$$

hence $\rho$ is always strongly convex by Proposition 1, and

$$
\begin{aligned}
\mathscr{C}_{o}^{*} & =\mathbb{R}_{\geq 0}(l, 1)+\mathbb{R}_{\geq 0}(-k, 1) \\
\Delta\left(L^{*}\right)_{o} & =[-k, l] .
\end{aligned}
$$

The bundle $\mathscr{O}\left(\iota^{*} \rho\right)$ is the ample line bundle $\mathfrak{O}_{\mathbb{P}^{1}}(k+l)$. The map $p_{\rho}$ of $(20)$ is given by

$$
p_{\rho}: D \rightarrow \bar{L},\left(m_{1}, m_{2}\right) \mapsto\left(m_{1}-m_{2}, k m_{1}+l m_{2}\right),
$$

whose cokernel is generated by $q_{L}$ with $(k+l) q_{L}=p_{o}(1,1)$. The dual map $p_{\rho}^{*}$ becomes :

$$
q_{\rho}^{*}: \bar{L}^{*}\left(=\mathbb{Z}^{2}\right) \rightarrow D^{*}\left(=\mathbb{Z}^{2}\right),(b, a) \mapsto(a k+b, \mathrm{a} l-b)
$$

$p_{\rho}$ induces an isomorphism between the first quadrant cone in $D_{\mathbb{R}}$ and the cone $\mathscr{C}_{\rho}$ in $\bar{L}_{\mathbb{R}}$. Hence the space $E_{\rho}^{*}$ is the quotient of $\mathbb{C}^{2}-\{0\}$ by the multiplication $\omega$ (=the primitive $(k+l)$-th root of unity), and the bundle map induced by $p_{\rho}$ is the projection

$$
\mathbb{C}^{2}-\{0\} \rightarrow\left(\mathbb{C}^{2}-\{0\}\right) /\langle\omega\rangle=E_{\rho}^{*}
$$

The basis of $\Gamma\left(\mathbb{P}_{(\Sigma, L)}, \mathscr{O}\left(\iota^{*} \rho\right)\right)$ in Proposition 2 consists of

$$
(m, 1), \quad m \in \mathbb{Z} \cap[-k, l],
$$


bijective to the following elements under $p_{\rho}^{*}$ :

$$
\left(m_{1}, m_{2}\right) \in \mathbb{Z}_{\geq 0}^{2}, \quad m_{1}+m_{2}=k+l
$$

This gives the usual monomial polynomial basis for $\Gamma\left(\mathbf{P}^{1}, \mathscr{O}(k+l)\right)$. As a consequence, for a general hypersurface $X$ of $\mathbb{P}_{(\Sigma, L)}$ defined by a section of $\mathscr{O}\left(\iota^{*} \rho\right)$, the equality

$$
|X|=\left|L^{*} \cap \operatorname{Int}\left(\Delta\left(L^{*}\right)_{\rho}\right)\right|+1
$$

holds.

(II) The weighted projective $n$-space $\mathbf{P}_{\left(n_{t}\right)}^{n}$ with weights $n_{i}, 1 \leq i \leq n+1$, satisfying $\operatorname{gcd}\left(n_{\jmath} \mid j \neq i\right)=1$ for all $i$. Now $L$ is the $n$-dimensional lattice generated by $n+1$ elements $\delta_{j}, 1 \leq j \leq n+1$, with the only relation

$$
\sum_{j=1}^{n+1} n_{j} \delta_{j}=0
$$

Set

$$
\begin{aligned}
& D=\mathbb{Z}^{n+1}, \\
& \beta: D \rightarrow L,\left(m_{1}, \ldots, m_{n+1}\right) \mapsto \sum_{j=1}^{n+1} m_{j} \delta_{j} . \\
& \sum=\left\{\sum_{j \in I} \mathbb{R}_{\geq 0} \delta_{j}|I \subset\{1, \ldots, n+1\},| I \mid \leq n\right\}
\end{aligned}
$$

We have

$$
\begin{aligned}
\mathbf{n} & =\mathbb{Z}\left(n_{1}, \ldots, n_{n+1}\right) \\
\sum^{1} & =\left\{\delta_{j}\right\}_{j=1}^{n+1}, \\
\mathbb{P}_{(\Sigma, L)} & =\mathbb{P}_{\left(n_{\imath}\right)}^{n},
\end{aligned}
$$

and $(7)$ is the natural projection:

$$
\pi: \mathbb{C}^{n+1}-\{0\} \rightarrow \mathbb{P}_{\left(n_{t}\right)}^{n},\left(z_{1}, \ldots, z_{n+1}\right) \mapsto\left[z_{1}, \ldots, z_{n+1}\right]
$$

Let us make the following identifications :

$$
D^{*}=\mathbb{Z}^{n+1}, L^{*}=\left\{\left(k_{1}, \ldots, k_{n+1}\right) \in D^{*} \mid \sum_{j} n_{j} k_{j}=0\right\}
$$


For

$$
\rho=\left(\rho_{1}, \ldots, \rho_{n+1}\right) \in D^{*}-\{0\}, \quad \rho_{j} \in \mathbb{Z}_{\geq 0}
$$

the linear map

$$
p_{\rho}: D\left(=\mathbb{Z}^{n+1}\right) \rightarrow \bar{L},\left(m_{1}, \ldots, m_{n+1}\right) \mapsto\left(\sum_{j} m_{j} \delta_{j}, \sum_{j} m_{j} \rho_{j}\right)
$$

induces an isomorphism between the first quadrant cone in $D_{\mathbb{R}}$ and the cone $\mathscr{C}_{\rho}$ in $\bar{L}_{\mathbf{R}}$. Hence $\rho$ is strictly convex by Proposition 1 . It is easy to see the relation

$$
q_{L}=\sum_{j=1}^{n+1} r_{j}\left(\rho_{j}, \delta_{j}\right), \quad r_{j}:=\frac{n_{j}}{\sum_{k=1}^{n+1} n_{k} \rho_{k}}
$$

holds in $\bar{L}_{Q}$. The affine variety $A_{\rho}$ of (30) is isomorphic to the quotient of $\mathbb{C}^{n+1}$ by the cyclic group generated by a diagonal element dia. $\left[r_{1}, \ldots, r_{n+1}\right]$. One can easily see that

$$
\begin{gathered}
\mathscr{C}_{\rho}^{*}=\sum_{j=1}^{n+1} \mathbb{R}_{\geq 0}\left(\left(a_{j, 1}, \ldots, a_{j, n+1}\right), 1\right), a_{j, i}= \begin{cases}-\rho_{\imath}, & \text { for } i \neq j, \\
\frac{1}{n_{j}} \sum_{k \neq \rho} \rho_{k} n_{k}, & \text { for } i=j\end{cases} \\
\Delta\left(L^{*}\right)_{\rho}=\text { the convex hull spanned by }\left(a_{j, 1}, \ldots, a_{j, n+1}\right), 1 \leq j \leq n+1 .
\end{gathered}
$$

Therefore the integral condition for $\Delta\left(L^{*}\right)_{\rho}$ is equivalent to

$$
n_{j} \mid \sum_{k=1}^{n+1} \rho_{k} n_{k} \text { for all } j
$$

(III) Toric variety which dominates $\mathbb{P}_{\left(n_{t}\right)}^{n} / G$ with $G$ a finite diagonal linear group of $\mathbb{C}^{n+1}$. Consider $\mathbb{C}^{* n+1}$ as the diagonal group and denote

$$
\exp : \mathbb{R}^{n+1} \rightarrow \mathbb{C}^{* n+1},\left(x_{1}, \ldots, x_{n+1}\right) \mapsto\left(e^{2 \pi i x_{1}}, \ldots, \mathrm{e}^{2 \pi i x_{n+1}}\right)
$$

$\exp ^{-1}(G)$ is a lattice in $\mathbb{R}^{n+1}$ containing the standard one $\mathbb{Z}^{n+1}$, and $\exp ^{-1}(G) \cap$ $\mathbb{Q}\left(n_{1}, \ldots, n_{n+1}\right)$ is a 1 -dimensional lattice with the generator $\left(q_{1}, \ldots, q_{n+1}\right)$. Let $L$ be the $n$-dimension lattice defined by

$$
L:=\exp ^{-1}(G) / \mathbb{Z}\left(q_{1}, \ldots, q_{n+1}\right)
$$


and $\delta_{j}, 1 \leq j \leq n+1$, be the elements in $L$ corresponding to the standard basis of $\mathbb{Z}^{n+1}$. Define the fan $\Sigma_{0}$ in $L_{\mathbf{R}}$ by

$$
\sum_{0}=\left\{\sum_{j \in J} \mathbf{R}_{\geq 0} \delta,|J \subset\{1, \ldots, n+1\},| J \mid \leq n\right\} .
$$

Then $\mathbb{P}_{\left(n_{t}\right)}^{n} / G$ is the toric variety $\mathbb{P}_{\left(\Sigma_{0}, L\right)}$. A refinement $\Sigma$ of $\sum_{0}$ gives rise a toric morphism

$$
\mathbb{P}_{(\Sigma, L)} \rightarrow \mathbb{P}_{\left(\Sigma_{0}, L\right)}
$$

Irreducible toric divisors of $\mathbb{P}_{\left(\Sigma_{0}, L\right)}$ are parametrized by

$$
\sum_{0}^{1}=\left\{\delta_{1}, \ldots, \delta_{n+1}\right\}
$$

and they give rise to $n+1$ irreducible toric divisors in $\mathbb{P}_{(\Sigma, L)}$, in which the rest ones are given by $e_{\delta}, \delta \in \Sigma^{1}-\sum_{0}^{1}$. For $\delta \in \Sigma^{1}-\Sigma_{0}^{1}$ one has the expression

$$
\delta=\sum_{j=1}^{n+1} a_{\delta, j} \delta_{j}, a_{\delta, j} \in \mathbf{Q}
$$

Then the subspace $\mathbb{n}_{\mathbf{C}}$ of $D_{\mathbf{C}}$ is generated by the following elements :

$$
\sum_{j=1}^{n+1} q_{j} e_{\tilde{\delta},} ; e_{\tilde{\delta}}-\sum_{j=1}^{n+1} a_{\tilde{\delta}, j} e_{\delta}, \delta \in \Sigma^{1}-\sum_{0}^{1},
$$

which form a basis of $\mathbf{n}_{\mathbf{C}}$ indexed by $\{0\} \cup \Sigma^{1}-\sum_{0}^{1}$. Hence one has the following isomorphic vector spaces:

$$
\operatorname{Pic}\left(\mathbf{P}_{(\Sigma, L)}\right)_{\mathbf{C}} \simeq \mathbf{n}_{\mathbf{C}}^{*} \simeq \bigoplus_{z \in\{0\} \cup \Sigma^{1}-\Sigma_{0}^{1}} \mathrm{C}_{z}
$$




\section{\$6. Rational Picard Groups of Hypersurfaces}

Now we are going to determine the rational Picard groups of hypersurfaces in a toric varieties. The non-ample hypersurfaces will be our main concern here. For the rest of this paper, we shall always assume the dimension of $\mathbb{P}_{(\Sigma, L)}$ is at least 4 , i.e.

$$
n \geq 4 \text {. }
$$

First we consider the case when $\mathscr{C}_{\rho}$ is strongly convex, (a weaker condition than the ampleness of $\left.\mathscr{O}\left(\iota^{*} \rho\right)\right)$. A similar conclusion for the second cohomology in Proposition 3 holds for this situation, which was stated in [8] without the proof. Here we present a detailed argument.

Proposition 4. Let $\rho$ be an element in $D^{*}$ such that $\mathscr{C}_{\rho}$ is a strongly convex cone with $\left\{\left(\delta, \rho_{\delta}\right) \mid \delta \in \sum^{1}\right\}$ as the fundamental generators. Let $X$ be a quasi-smooth hypersurface of $\mathbb{P}_{(\Sigma, L)}$ defined by a section of $\mathbb{O}\left(\iota^{*} \rho\right)$. Then the following vector spaces are naturally isomorphic:

$$
H^{2}\left(\mathbb{P}_{(\Sigma, L)}, \mathbb{C}\right) \simeq H^{2}(X, \mathbb{C}) \simeq \operatorname{Pic}(X)_{\mathbf{C}}
$$

Consequently the inclusion map induces the isomorphism:

$$
\operatorname{Pic}\left(\mathbb{P}_{(\Sigma, L)}\right)_{\mathrm{C}} \simeq \operatorname{Pic}(X)_{\mathrm{C}}
$$

Proof. Let $U$ be the complement of $X$ in $\mathbb{P}_{(\Sigma, L)}$. By the argument in Proposition 3, we have the exact sequence (33) and the isomorphism (31). For the first isomorphism of the conclusion, it suffices to show the vanishing of the following cohomology (with complex coefficients throughout):

$$
H^{2 n-3}(U)=H^{2 n-2}(U)=0 .
$$

Let $\Sigma_{0}$ be the complete fan in $L_{\mathbb{R}}$ obtained by the projection of proper faces of $\mathscr{C}_{\rho}$. Note that $\Sigma_{0}$ may not be simplical in general, while $\Sigma$ is a simplicial refinement $\Sigma_{0}$ with $\sum_{0}^{(1)}=\Sigma^{(1)}$. Hence one has the equivariant morphism :

$$
\phi: \mathbb{P}_{(\Sigma, L)} \rightarrow \mathbb{P}_{(\Sigma 0, L)}
$$

which induces the isomorphic toric divisor-groups. The graph of $f_{\rho}$ is linear on each polyhedral cone in $\sum_{0}$. By the strong convexity of $\mathscr{C}_{\rho}, \rho$ determines an ample toric divisor in $\mathbb{P}_{\left(\Sigma_{0}, L\right)}$, whose pull-back under the map $\phi$ equals to the 
bundle $\mathcal{O}\left(\iota^{*} \rho\right)$ over $\mathbb{P}_{(\Sigma, L)}$. As they have the same global sections, there is an ample hypersurface $X_{0}$ of $\mathbf{P}_{\left(\Sigma_{0}, L\right)}$ with $X=\phi^{-1}\left(X_{0}\right)$. Denote

$$
U_{0}:=\mathbb{P}_{\left(\Sigma_{0}, L\right)}-X_{0}
$$

then $U_{0}$ is an affine variety with $\phi^{-1}\left(U_{0}\right)=U$ and $H^{j}\left(U_{0}\right)=0$ for $j>n$. Since $\left(\delta, \rho_{\delta}\right), \delta \in \Sigma^{1}$, are the fundamental generators of $\mathscr{C}_{\rho}$, the exceptional set of $\phi$ is of codimension greater than one. There exists an analytic subspace $V_{0}$ of $U_{0}$ such that $V\left(:=\phi^{-1}\left(V_{0}\right)\right)$ is of codimension $\geq 2$ and

$$
U-V \stackrel{\phi}{\simeq} U_{0}-V_{0}
$$

For a suitable "tubular" neighborhood $T$ of $V$ in $U$, and $T^{\circ}=T-V$, we have the following commutative Mayer-Vietoris exact sequences:

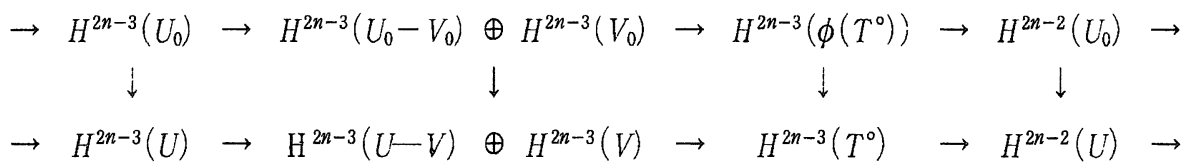

Since

$$
H^{\jmath}\left(U_{0}\right)=H^{\jmath}\left(V_{0}\right)=H^{j}(V)=0, \text { for } j \geq 2 n-3
$$

we obtain (36), hence the isomorphism between $H^{2}\left(\mathbf{P}_{(\Sigma, L)}, \mathbb{C}\right)$ and $H^{2}(X, \mathbf{C})$ Next we are going to show the vanishing property of the cohomology groups,

$$
H^{1}(X, \mathscr{O})=H^{2}(X, \mathscr{O})=0
$$

which implies the isomorphism between $H^{2}(X, \mathbf{C})$ and $\mathrm{Pic}(X)$ c. Let $L_{1}$ be a sublattice of $L$ such that the pull-back of $X$, denoted by $X_{1}$, is Cartier under the finite abelian cover :

$$
\mathbf{P}_{\left(\Sigma, L_{1}\right)} \rightarrow \mathbf{P}_{(\Sigma, L)}
$$

Now it suffices to show the vanishing of $H^{2}\left(X_{1}, \mathscr{O}\right)$, for $i=1,2$. By replacing $X_{1}, L_{1}$ by $X$ and $L$, we may assume the hypersurface $X$ to be Cartier in $\mathbf{P}_{(\Sigma, L)}$, but with no quasi-smooth condition required, for the purpose of vanishing property of $H^{i}(X, \mathbb{O})$ for $i=1,2$. By the cohomology sequence associated to the exact sequence of sheaves over $\mathbb{P}_{(\Sigma, L)}$ : 


$$
0 \rightarrow \mathscr{O}_{\mathbb{P}_{(\Sigma, L)}}(-X) \rightarrow \mathscr{O}_{\mathbb{P}_{(\Sigma, L)}} \rightarrow \mathscr{O}_{X} \rightarrow 0
$$

and the well-known fact

$$
H^{\jmath}(\mathbb{P}(\Sigma, L), \mathscr{O})=0 \text { for } j>0
$$

the vanishing of $H^{i}(X, \mathfrak{O}),(i=1,2)$ follows from

$$
H^{i}\left(\mathbb{P}_{(\Sigma, L)}, \mathfrak{O}_{\mathbb{P}_{(\Sigma, L)}}(-X)\right)=0 \text { for } \quad j=2,3
$$

Note that $\mathscr{O}_{\mathbb{P}_{(\Sigma, L)}}(-X)$ is the sheaf of sections for the line bundle $\mathscr{O}\left(-\iota^{*} \rho\right)$. By Serre duality, $H^{\jmath}\left(\mathbb{P}_{(\Sigma, L)}, \mathcal{O}_{\mathbb{P}_{(\Sigma, L)}}(-X)\right)$ is dual to $H^{n-j}\left(\mathbb{P}_{(\Sigma, L)}, \omega_{\mathbb{P}_{(\Sigma, L)}}(X)\right)$, where $\omega_{\mathbb{P}_{(\Sigma, L)}}$ is the canonical sheaf of $\mathbb{P}_{(\Sigma, L)}$. Under the map $\phi$ of $(37)$, we have

$$
\omega_{\mathbb{P}_{(\Sigma, L)}}(X)=\phi^{*}\left(\omega_{\mathbb{P}_{(\Sigma, L)}}\left(X_{0}\right)\right)
$$

Since $X_{0}$ is an ample divisor in $\mathbb{P}_{\left(\Sigma_{0}, L\right)}$,

$$
H^{k}\left(\mathbb{P}_{\left(\Sigma_{0}, L\right)}, \omega_{\mathbb{P}_{(\Sigma 0, L)}}\left(X_{0}\right)\right)=0 \text { for } k>0
$$

By $n \geq 4$ and

$$
R_{\phi *}^{k} \mathscr{O}_{\mathbb{P}_{(\Sigma, L)}}=0 \text { for } k>0
$$

one has

$$
H^{n-\jmath}\left(\mathbb{P}_{(\Sigma, L)}, \omega_{\mathbb{P}_{(\Sigma, L)}}(X)\right) \simeq H^{n-j}\left(\mathbb{P}_{\left(\Sigma_{0}, L\right)}, \omega_{\mathbb{P}_{(\Sigma, L)}}\left(X_{0}\right)\right)=0 \quad \text { for } \quad j=2,3
$$

This completes the proof of this proposition.

We now generalize Proposition 4 to a larger class of hypersurfaces in toric varieties.

Theorem 2. Let $X$ be a quasi-smooth hypersurface of $\mathbb{P}(\Sigma, L)$ defined by a section $s$ of $\mathcal{O}\left(c^{*} \rho\right)$ for a convex element $\rho$ in $D^{*}$. Let $\sum(\rho)$ be the fan in $\bar{L}_{\mathbb{R}}$ defined by (16). Assume the Newton polygon of $s$ is equal to $\Delta\left(L^{*}\right)_{\rho}$, and $\sum(\rho)$ satisfies the following conditions:

(i)

$$
\sum(\rho)^{(1)} \cap \bigcup_{F: \text { facet of } \mathscr{C}_{o}} \operatorname{Int}(F)=\emptyset .
$$


(ii) $\sum(\rho)$ is a refinement of some fan $\Lambda$ in $\bar{L}_{\mathbf{R}}$ with:

$$
|\Lambda|=\partial \mathscr{C}_{\rho}, \quad \Lambda^{(1)}=\left\{1-\text { face of } \mathscr{C}_{\rho}\right\} .
$$

Then we have the isomorphism of vector spaces:

$$
\begin{aligned}
\operatorname{Pic}(X)_{\mathbf{C}} \simeq \operatorname{Pic}\left(\mathbb{P}_{(\Sigma, L)}\right)_{\mathbf{c}} \oplus \bigoplus_{\tau, z}\left\{\mathbf{C}_{z} \mid z \in\right. & \left.(\operatorname{Int}(\underline{\tau}) \cap \Delta(L) \cap L) \times\left(\operatorname{Int}\left(\underline{\tau^{\prime}}\right) \cap \Delta\left(L^{*}\right)_{\rho} \cap L^{*}\right)\right\},
\end{aligned}
$$

where the index $\tau$ runs over the codimensional 2 faces of $\mathscr{C}_{\rho}$ with its dual $\tau^{\prime}$ in $\mathscr{C}_{\rho}^{*}$, $\underline{\tau}, \tau^{\prime}$ mean the projection on $L_{\mathbf{R}}, L_{\mathbf{R}}^{*}$ respectively, and $\Delta(L), \Delta\left(L^{*}\right)_{\rho}$ are defined by (1) (24).

Proof. Let $\Sigma_{0}$ be the complete fan in $L_{\mathbf{R}}$ obtained by the projection of $\Lambda$. Then $\Sigma$ is a refinement of $\Sigma_{0}$, and one has the $\mathbf{T}(L)$-morphism,

$$
\varphi: \mathbb{P}_{(\Sigma, L)} \rightarrow \mathbb{P}_{(\Sigma, L)},
$$

with the relation of toric divisors given by

$$
\sum^{1} \supset \sum_{0}^{1}
$$

Let $\eta$ by the element in $D\left(\Sigma_{0}, L\right)_{\mathbf{Q}}^{*}$ defined by

$$
\eta=\sum_{\delta \in \Sigma_{0}^{1}} \eta_{\delta} e_{\delta}, \eta_{\delta}:=\rho_{\delta} \quad \text { for } \quad \delta \in \sum_{0}^{1}
$$

The cones $\mathscr{C}_{\rho}$ and $\mathscr{C}_{\eta}$ are the same in $L_{\mathbf{R}}$, which implies

$$
\rho=\varphi^{*}(\eta)
$$

as divisors in $\mathbf{P}_{(\Sigma, L)}$. Hence there exists a quasi-smooth hypersurface $X_{0}$ in $\mathbb{P}_{\left(\Sigma_{0}, L\right)}$ with $\varphi^{*}\left(X_{0}\right)=X$. The Newton polygon for the equation of $X_{0}$ equals to $\Delta\left(L^{*}\right)_{\eta}$, which is the same as $\Delta\left(L^{*}\right)_{\rho}$. Since the Picard groups of $\mathbf{P}_{(\Sigma, L)}$ and $X$ are generated by divisors, hence one has the following identification of vector spaces :

$\operatorname{Pic}(X)_{\mathbf{c}}=\operatorname{Pic}\left(X_{0}\right)_{\mathbf{c}} \oplus \oplus\left\{\mathbf{C}_{e} \mid e\right.$ :irreducible exceptional divisor in $X$ over $\left.X_{0}\right\}$ $\operatorname{Pic}\left(\mathbf{P}_{(\Sigma, L)}\right) \mathbf{C}=\operatorname{Pic}\left(\mathbb{P}_{(\Sigma, L)}\right)_{\mathbf{C}} \oplus$ $\bigoplus\left\{\mathbf{C} E \mid E\right.$ : irreducible exceptional divisor in $\mathbf{P}_{(\Sigma, L)}$ over $\left.\mathbf{P}_{(\Sigma, L)}\right\}$. 
In the second equality above, the right hand side is a vector space with a basis parametrized by $\sum^{1}$. By Proposition 4 , we have

$$
\operatorname{Pic}\left(X_{0}\right)_{\mathrm{C}}=\operatorname{Pic}\left(\mathbb{P}_{\left(\Sigma_{0}, L\right)}\right)_{\mathrm{C}}
$$

Let orb $(\lambda)$ be $\mathbb{T}(L)$-orbit in $\mathbb{P}_{\left(\Sigma_{0}, L\right)}$ for $\lambda \in \Sigma_{0}$. The closure $\overline{\text { orb }(\lambda)}$ of orb $(\lambda)$ is a toric variety having the lattice and the fan given by

$$
L / \mathbb{Z}(L \cap \lambda), \quad \operatorname{Star}(\lambda):=\left\{\bar{\sigma} \mid \sigma \in \sum_{0}, \lambda \prec \sigma\right\},
$$

where $\bar{\sigma}$ denotes the image of $\sigma$ in $L_{\mathbb{R}} / \mathbb{R} \lambda$. Hence $\operatorname{dim} \overline{\operatorname{orb}(\lambda)}+\operatorname{dim}(\lambda)=n$. Every element $\lambda$ in $\Lambda$ is contained in an unique face $\tau(\lambda)$ of $\mathscr{C}_{\rho}$ with the same dimension. One has

$$
\mathscr{C}_{\rho}^{*} \cap \lambda^{\perp}=\tau(\lambda)^{\prime}\left(=\mathscr{C}_{\rho}^{*} \cap \tau(\lambda)^{\perp}\right)
$$

Since the Newton polygon for $X_{0}$ is equal to $\Delta\left(L^{*}\right)_{\rho}$, together with the equality of (34) for each 1-dimensional $\overline{\operatorname{orb}(\lambda)}$, one concludes the following relation holds :

$X_{0} \bigcap \overline{\operatorname{orb}(\lambda)}=\left\{\begin{array}{lr}\emptyset, & \text { if } \operatorname{dim} \overline{\operatorname{orb}(\lambda)}=0, \\ \text { a finite set with }\left|\operatorname{Int}\left(\tau(\lambda)^{\prime}\right) \cap \Delta\left(L^{*}\right)_{\rho} \cap L^{*}\right|+1 \text { elements, } & \text { if } \operatorname{dim} \overline{\operatorname{orb}(\lambda)}=1, \\ \text { a connected set, } & \text { if } \operatorname{dim} \overline{\operatorname{orb}(\lambda)} \geq 2 .\end{array}\right.$

By the condition (i) in our assumption and (38), we obtain the result.

\section{\$7. Amti-Canonical Hypersurfaces and Callabi-Yau 3-Folds}

In this section, we are going to apply our previous results to the discussion of an anti-canonical hypersurface $X$ in $\mathbb{P}_{(\Sigma, L)}$. Note that such $X$ has the trivial canonical sheaf. As the canonical sheaf of $\mathbb{P}_{(\Sigma, L)}$ is given by $\iota^{*} \kappa$ for $\kappa$ defined in (13), we now set the element $\rho$ of $D^{*}$ in Section 6 equal to $-\kappa$ for the discussion of this section. By the corollary of Proposition 1, the convexity of $-\kappa$ is equivalent to the convex property of the polytope $\Delta(L)$ in $L_{\mathbb{R}}$. In this situation, $\Delta(L)$ is integral with respect to $L$, and $\Delta\left(L^{*}\right)_{-\varkappa}$ is the dual polytope of $\Delta(L)$ in $L_{\mathbb{R}}^{*}$ defined by

$$
\Delta(L)^{*}:=\left\{y \in L_{\mathbb{R}}^{*} \mid\langle x, y\rangle \geq-1 \forall x \in \Delta(L)\right\}
$$


If the Newton polygon of a section of $\mathscr{O}\left(-\iota^{*} \kappa\right)$ is equal to $\Delta\left(L^{*}\right)_{-\kappa},(\Delta(L), L)$ forms a reflexive polytope in the sense of Batyrev [4]:

Definition 4. Let $L$ be a lattice, and $\Delta$ a convex polytope in $L_{\mathbf{R}}$. $(\Delta, L)$ is called a reflexive polytope if both $\Delta$ and $\Delta^{*}$ are integral.

Conversely, for a toric variety $\mathbf{P}_{(\Sigma, L)}$ with the reflexive polytope $(\Delta(L), L)$, $-\kappa$ is an element in $D^{*}$. Consider a hypersurface $X$ of $\mathbf{P}_{(\Sigma, L)}$ with $\omega_{X}=\mathscr{O}_{X}$, or equivalently to say, $X$ is defined by a section of $\mathscr{O}\left(-\iota^{*} \kappa\right)$. As a corollary of Theorem 1 and 2, one has the following result :

Proposition 5. For $n \geq 4$, let $\mathbb{P}_{(\Sigma, L)}$ be a $n$-dimensional toric variety with $(\Delta(L), L)$ being a reflexive polytope. Let $X$ be a quasi-smooth hypersurface of $\mathbf{P}_{(\Sigma, L)}$ defined by a section in $\mathcal{O}\left(-c^{*} \kappa\right)$ whose Newton polygon equals to $\Delta(L)$. Assuming

(i)

$$
\sum^{1} \cap \bigcup_{F: \text { facet of } \Delta(L)} \operatorname{Int}(F)=\emptyset .
$$

(ii) $\sum$ is a refinement of some fan $\sum_{0}$ in $L_{\mathbb{R}}$ with $\sum_{0}^{1}=\{$ vertex of $\Delta(L)\}$. Then we have the isomorphic vector spaces:

$$
\begin{aligned}
\operatorname{Pic}(X)_{\mathbf{C}} \simeq \mathbf{n}\left(\sum, L\right)_{\mathbf{C}}^{*} \oplus & \bigoplus_{F, z}\left\{\mathbf{C}_{z} \mid z \in\right. \\
& \left.(\operatorname{Int}(F) \cap \Delta(L) \cap L) \times\left(\operatorname{Int}\left(F^{\prime}\right) \cap \Delta(L)^{*} \cap L^{*}\right)\right\},
\end{aligned}
$$

where the index $F$ runs over the codimensional 2 faces of $\Delta(L)$ with its dual face $F^{\prime}$ defined by

$$
F^{\prime}:=\left\{y \in \Delta(L)^{*} \mid\langle x, y\rangle=-1 \text { for } x \in F\right\}
$$

For $n=4$, the hypersurface $X$ in the above proposition is smooth under some "maximal" condition on $\Sigma^{1}$.

Proposition 6. Let $X$ be a hypersurface of $\mathbf{P}_{(\Sigma, L)}$ in Proposition 5 for $n=4$, and assume

$$
\Sigma^{1}=(L \cap \partial \Delta(L))-\bigcup_{F: \text { facet of } \Delta(L)} \operatorname{Int}(F) .
$$


Then $X$ is a smooth CY space.

Proof. As in the proof of Theorem 2, we consider the morphism $\varphi$ from $\mathbb{P}_{(\Sigma, L)}$ to $\mathbb{P}_{\left(\Sigma_{0}, L\right)}$, and the quasi-smooth hypersurface $X_{0}$ of $\mathbb{P}_{\left(\Sigma_{0}, L\right)}$ with $X=\varphi^{*}\left(X_{0}\right)$. Now $X_{0}$ has the trivial canonical sheaf and the Newton polygon of its equation equals to $\Delta(L)^{*}$. Given an element $p$ in $X_{0}$, we are going to show that $X$ is smooth near $\varphi^{-1}(p)$. Let $\lambda$ be the element in $\Sigma_{0}$ such that the $\mathbb{T}(L)$-orbit $\operatorname{orb}(\lambda)$ in $\mathbb{P}_{(\Sigma, L)}$ contains $p$. From the assumption on $\Sigma_{0}$ and $\sum$, it needs only to consider the case when $m:=\operatorname{dim}$ orb $(\lambda)=2,3$. Let $\sigma$ be a 3 -dimensional simplicial cone in $\sum_{0}$ which contains $\lambda$ as a proper face. $\sigma$ is generated by the vertices $\delta_{i}, 1 \leq i \leq 4$, of $\Delta(L)$, and one may assume

$$
\sigma=\sum_{j=1}^{4} \mathbb{R}_{\geq 0} \delta_{j}, \quad \lambda=\sum_{j=1}^{m} \mathbb{R}_{\geq 0} \delta_{j}
$$

Associated to $\sigma$, there is the affine open subset $U_{\sigma}$ of $\mathbb{P}_{\left(\Sigma_{0}, L\right)}$ with a finite abelian cover (27):

$$
\psi: \mathbb{C}^{4} \rightarrow U_{\sigma}=\mathbb{C}^{4} / I_{\sigma}, U_{\sigma}:=\operatorname{Spec} \mathbb{C}\left[\check{\sigma} \cap L^{*}\right], I_{\sigma}:=L / \sum_{i=1}^{4} \mathbb{Z} \delta_{i}
$$

Let $\left(t_{1}, t_{2}, t_{3}, t_{4}\right)$ be the coordinates of $\mathbb{C}^{4}$ corresponding to the dual basis $\left\{\delta_{i}^{*}\right\}_{i=1}^{4}$. By $(29), \phi^{*}\left(X_{0} \cap U_{\sigma}\right)$ is a non-singular hypersurface in $\mathbb{C}^{4}$ defined by a $I_{\sigma}$-invariant polynomial :

$$
\eta(t)=\sum_{y \in L^{*} \cap \Delta(L)^{*}} \alpha_{y} t^{k(y)}, \quad t^{k(y)}:=\prod_{i=1}^{4} t_{i}^{k(y)}, \quad k(y)_{i}:=\left\langle\delta_{l}, y\right\rangle+1
$$

Let $y_{0}$ be the vertice of $\Delta(L)^{*}$ dual to the facet of $\Delta(L)$ containing all $\delta_{i}$ 's. Since the Newton polygon for $X_{0}$ is equal to $\Delta(L)^{*}, y_{0}$ belongs to $L^{*}$ and

$$
\alpha_{y_{0}} t^{k\left(y_{0}\right)}=\alpha_{y_{0}} \neq 0
$$

In the case for $m=3$, by Lemma 4 and the condition (i) of the assumption, $\delta_{1}$, $\delta_{2}, \delta_{3}$, together with some vertex $\delta_{4}^{\prime}$ of $\Delta(L)$ not equal to $\delta_{4}$, forms a 3 -dimensional element in $\sum_{0}$, hence its dual vertex $y_{1}$ in $\Delta(L)^{*}$ is not equal to $y_{0}$. The same conclusion holds also for the case $m=2$ if one starts with a suitable choice of $\delta_{i}, 1 \leq i \leq 4$. Therefore we have 


$$
\alpha_{y_{1}} t^{k\left(y_{1}\right)}=\alpha_{y_{1}} t_{4}^{k_{4}}, k_{4} \geq 2
$$

and the projection to the first three components $\left(t_{1}, t_{2}, t_{3}\right)$ defines the local coordinate system of $\phi^{*}\left(X_{0} \cap U_{\sigma}\right)$ near $\phi^{-1}(p)$. Through the functions $t_{1}, t_{2}, t_{3}$ and $f(t)$, one has the isomorphims:

$$
\left(\mathbf{P}_{\left(\Sigma, L_{0}\right)}, p\right) \simeq\left(\mathbf{C}^{3} / G, 0\right) \times(\mathbf{C}, 0) \simeq\left(X_{0}, p\right) \times(\mathbf{C}, 0)
$$

where $G$ is a finite diagonal subgroup of $S L_{3}(\mathbb{C})$ (by the trivial canonical sheaf of $X_{0}$ ). The group of $G$ is described by

$$
G \simeq\left(L \cap \sum_{j=1}^{m} \mathbb{R} \delta_{j}\right) / \sum_{j=1}^{m} \mathbb{Z} \delta_{j}
$$

Denote $\left\langle\delta_{\jmath}\right\rangle_{j=1}^{m}$ the convex set spanned by $\left\{\delta_{j}\right\}_{j=1}^{m}$. Since $G$ is a subgroup of $S L_{m}(\mathbf{C})$ for $m=2,3, L \cap \sum_{j=1}^{m} \mathbb{R} \delta$, is a sublattice of $L$ generated by $L \cap$ $\left\langle\delta_{j}\right\rangle_{j=1}^{m}$, whose classes generate the group $G$. The fan $\sum$ induces a triangulation of $\left\langle\delta_{j}\right\rangle_{j=1}^{m}$ with $L \cap\left\langle\delta_{j}\right\rangle_{j=1}^{m}$ as the set of vertices, hence it gives a crepant toric resolution of $\mathbb{C}^{3} / G$ [13] [20]. By (39), both $\mathbf{P}_{(\Sigma, L)}$ and $X$ are non-singular near $\phi^{-1}(p)$. Therefore we obtain the result of this proposition.

Remark. When the polytope is a 4-dimensional simplex, the concept of reflexive simplex $(\Delta, L)$ is equivalent to $\mathbb{P}_{\left(n_{i}\right)}^{4} / G$ in Sect. 5 (III) with $G \subset S L_{5}(\mathbb{C}$ ) and the "Fermat-type" condition on weights $n_{\imath}$ 's, (a precise statement see Appendix (II)). In this situation, Propositions 5 and 6 are given in [21] [22] [23].

\section{Appendix}

(I) Remark on Proposition 3. For a hypersurface $X$ of $\mathbf{P}_{(\Sigma, L)}$ in Proposition 3 , one has the isomorphic Hodge groups:

$$
H^{q}\left(X, \Omega_{X}^{p}\right) \simeq H^{q+1}\left(\mathbf{P}_{(\Sigma, L)}, \Omega_{\mathbf{P}_{(\Sigma, L)}^{p+1}}\right), \text { for } p+q \geq n
$$

hence

$$
H^{q}\left(X, \Omega_{X}^{p}\right)=0, \text { for } p+q \geq n, p \neq q \text {. }
$$


In fact, the spectral sequence

$$
E_{1}^{p q}=H^{q}\left(\mathbb{P}_{(\Sigma, L)}, \Omega_{\mathbb{P}_{(\Sigma, L)}^{p}}^{p}(\log X)\right) \Rightarrow H^{p+q}\left(\mathbb{P}_{(\Sigma, L)}-X, \mathbb{C}\right)
$$

degenerates at $E_{1}$-term [7] [25]. By (32), we have

$$
H^{q}\left(\mathbb{P}_{(\Sigma, L)}, \Omega_{\mathbb{P}_{(\Sigma, L)}}^{p}(\log X)\right)=0, \quad \text { for } p+q>n
$$

Then the conclusion follows from

$$
H^{q}\left(\mathbb{P}_{(\Sigma, L)}, \Omega_{\mathbb{P}_{(\Sigma, L)}}^{p}\right)=0, \quad \text { for } p \neq q
$$

and the cohmologoy sequence for the exact sequence of sheves:

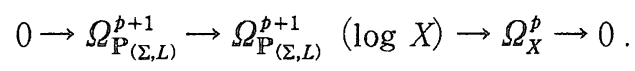

(II) Reflexive simplex. The following two sets are in one-one correspondence :

(i) The collection of all reflexive pair $(\Delta, M)$ with $\Delta$ a 4 -dimensional simplex.

(ii) The collection of all $\left(\mathbb{P}_{\left(n_{i}\right)}^{4}, G\right)$ with the weights $n_{i}$ 's and the group $G$ satisfying the conditions :

$$
d_{\jmath}:=\frac{d}{n_{j}} \in \mathbb{Z} \forall j, \quad d:=\sum_{i=1}^{n} n_{i}, \quad Q \subseteq G \subseteq S D
$$

where $Q$ is the group generated by dia. $\left[e^{2 \pi i / d 1}, \ldots, e^{2 \pi / / d 5}\right]$, and $S D=\left\{\right.$ dia. $\left[t_{1}, \ldots, t_{5}\right]$ $\in S L_{5}(\mathbb{C}) \mid t_{i}^{d_{i}}=1$ for all $\left.i\right\}$.

(For a proof of the above statement, see [4] [23])

\section{\$9. Acknowledgments}

Part of this work was done while the author was visiting Kyoto University and Tohoku University of Japan during the early spring of 1995. He wishes to thank Professors S. Mori, T. Oda and K. Saito for the kind invitations and warm hospitality. Especially to Professor T. Oda, the author was benefited a great deal from the many discussions, suggestions, and also the useful references related to this paper. 


\section{References}

[1] Andreotti, A. and Frankel. T., The Lefschetz theorem on hyperplane sections, Ann. of Math. 69 (1959), 713-717.

[2] Aspinwall, P. S., Greene, B. R. and Morrison, D. R., Calabi-Yau moduli space, mirror man ifolds and spacetime topology change in string theory, Nucl. Phys. B, 416 (1994), 414-480.

[3] Audin, M., The topology of torus actions on sympletic manifolds, Progr. Math., 93, Berlin, (1991).

[4] Batyrev, V. V., Dual polyhedra and mirror symmetry for Calabi-Yau hypersurfaces in toric varieties, J. Alg. Geom., 3 (1994), 493-535.

[5] Batyrev, V. V. and Cox, A. A., On the Hodge structure of projective hypersurfaces in toric varieties, Duke Math. J., 75 (1994), 293-338.

[6] Cox, D. A., The homogeneous coordinate ring of a toric variety, J. Alg. Geom., 4 (1995). 17-50.

[7] Danilov, V. I., The geometry of toric varieties, Russian Math. Surveys, 33, 2 (1978), 97-154.

[8] Danilov, V. I. and Khovankii A. G., Newton polyhedral and algorithm for computing Hodge-Deligne numbers. Math. USSR Izvestiya, 29 (1987), 279-298.

[9] Danilov, V. I., "De Rham complex on toridal variety" in Algebraic Geometry, ed. by I. Dolgachev and W. Fulton, Lecture Notes in Math., Springer-Verlag, Berlin, 1479 (1991), 26-38.

[10] Fulton, W., Introduction to toric varieties, Ann. of Math. Siud, 131. Princeton Univ. Press, 1993.

[11] Guillemin, V., Kaehler structures on toric varieties, J. Diff. Geom., 40 (1994), 285-309.

[12] Kempt, G., Knudson, F., Mumford. D. and Saint-Donat, Toroidal embedding 1. Lecture Notes in Math., Springer-Verlag, 339 (1973).

[13] Markushevich, D. G., Olshanetsky, M. A. and Perelomov. A. M., Description of a class of superstring compactifications related to semi-simple Lie algebra, Comm. Math. Phys., 111 (1987), 247-274.

[14] Manin, Y., and Tsfasman, M., Rational varieties: algebra, geometry, and arithmetıcs, Russian Math. Surveys, 41 (1986), 51-116.

[15] Oda, T., Lectures on torus embeddings and applications, Tata Inst of Fund. Res. Springer-Verlag, 58 (1978).

[16] - C Convex bodies and algebraic geometry-An introduction to the theory of toric varieties, Ergeb. Math. (3) , 15, Springer-Verlag, Berlin, Heidelberg, New York, London, Paris, Tokyo. 1988.

[17] - Geometry of toric varieties. Proc. of the Hyderabad Conf. on Algebraic Groups, Dec. 1989 (S. Ramanan, ed.) Manjo Prakashan, Madras, India.

[18] - Recent topics on toric varieties, Sugaku Expositions, to appear.

[19] Oda, T. and Park, H. S., Linear Gale transformations and Gelgand-Kapranov-Zelevinskij decompositions, Tohoku Math. J., 43 (1991), 375-399.

[20] Roan, S. S., On the generalization of Kummer surfaces, J. Diff. Geom., 30 (1989), 523-537.

[21] - The mirror of Calabi-Yau orbifolds, Internat. J. Math., (4) 2 (1991), 439-455.

[22] - The topological properties of Calabi-Yau mirror manifolds, Perspect. Math. phys. (R. Penner and S. T. Yau, ed.) International Press, (1994) 251-264.

[23] - Toric geometry in mirror geometry, Academia Sinica Math., preprint R940907-1.

[24] Roan. S. S. and Yau S. T., On Ricci flat 3-fold, Acta Math. Sinica, New Series (3), 3 (1987), 256-288. 
[25] Steenbrink, J. H., Mixed Hodge structure on the vanishing cohomology, in : Real and complex singularities (Proc. 9th Nordic summer school/NAVF Sympos. Math., Oslo 1976), 565-678. Sijthoff and Noordhoff, Alphen aan den Rijn 1977.

[26] Yau, S. T., (Ed.), Essays on mirror manifolds, International Press. Hong Kong, 1992. 